Draft Version November 8, 2018

Preprint typeset using $\mathrm{LAT}_{\mathrm{E}} \mathrm{X}$ style emulateapj v. 5/2/11

\title{
CONSTRAINING SATELLITE GALAXY STELLAR MASS LOSS AND PREDICTING INTRAHALO LIGHT I: FRAMEWORK AND RESULTS AT LOW REDSHIFT
}

\author{
Douglas F. Watson And Andreas A. Berlind ${ }^{1}$, \\ Department of Physics and Astronomy, Vanderbilt University, Nashville, TN 37235
}

ANDREW R. ZENTNER

Department of Physics and Astronomy \& Pittsburgh Particle physics, Astrophysics, and Cosmology Center (PITT PACC), The University of Pittsburgh, Pittsburgh, PA 15260

Draft version November 8, 2018

\begin{abstract}
We introduce a new technique that uses galaxy clustering to constrain how satellite galaxies lose stellar mass and contribute to the diffuse "intrahalo light" (IHL). We implement two models that relate satellite galaxy stellar mass loss to the detailed knowledge of subhalo dark matter mass loss. Model 1 assumes that the fractional stellar mass loss of a galaxy, from the time of merging into a larger halo until the final redshift, is proportional to the fractional amount of dark matter mass loss of the subhalo it lives in. Model 2 accounts for a delay in the time that stellar mass is lost due to the fact that the galaxy resides deep in the potential well of the subhalo and the subhalo may experience dark matter mass loss for some time before the galaxy is affected. We use these models to predict the stellar masses of a population of galaxies and we use abundance matching to predict the clustering of several $r$-band luminosity threshold samples from the Sloan Digital Sky Survey. Abundance matching assuming no stellar mass loss (akin to abundance matching at the time of subhalo infall) over-estimates the correlation function on small scales $(\lesssim 1 \mathrm{Mpc})$, while allowing too much stellar mass loss leads to an under-estimate of small-scale clustering. For each luminosity threshold sample, we are thus able to constrain the amount of stellar mass loss required to match the observed clustering. We find that satellite galaxy stellar mass loss is strongly luminosity dependent, with less luminous satellite galaxies experiencing substantially more efficient stellar mass loss than luminous satellites. With constrained stellar mass loss models, we can infer the amount of stellar mass that is deposited into the IHL. We find that both of our model predictions for the mean amount of IHL as a function of halo mass are consistent with current observational measurements. However, our two models predict a different amount of scatter in the IHL from halo to halo, with Model 2 being favored by observations. This demonstrates that a comparison to IHL measurements provides independent verification of our stellar mass loss models, as well as additional constraining power.
\end{abstract}

Subject headings: cosmology: theory — dark matter — galaxies: halos — galaxies: structure large-scale structure of universe

\section{INTRODUCTION}

In the concordance $\Lambda \mathrm{CDM}$ cosmology, galaxies, galaxy groups, and galaxy clusters form hierarchically. Highdensity regions condense and virialize, forming bound structures known as halos. Halos grow through the continual accretion of smaller objects. These accreted objects may survive within the virialized region of the primary halo as smaller, self-bound, orbiting dark matter clumps dubbed "subhalos" or "substructure" (Ghigna et al. 2000; Klypin et al. 1999; Diemand et al. 2004; Kravtsov et al. 2004a). Halos of sufficient mass are the natural sites of galaxy formation, with baryons cooling and condensing towards potential well minima (White \& Rees 1978; Blumenthal et al. 1986). When a halo is accreted by a larger halo, thus becoming a subhalo, the galaxy within it becomes a "satellite" galaxy within a group or cluster. Understanding the detailed relationship between (satellite) galaxies and (sub) halos is a long-standing focus of galaxy formation theory.

In the hierarchical paradigm, these smaller objects,

\footnotetext{
${ }^{1}$ Alfred P. Sloan Fellow
}

upon merging, become victims of intense tidal fields and interactions within the larger systems in which they reside. The dark matter mass associated with a subhalo may be rapidly stripped upon infall. This stripping acts on the periphery of the subhalo first, suggesting that the luminous galaxy, residing in the center of the subhalo, may be relatively unharmed. After enough time has elapsed, stripping of stars may begin to occur as well. These liberated stars that are ripped from galaxies are the likely source of "intrahalo light" (IHL: e.g., Gallagher \& Ostriker 1972; Merritt 1983; Bvrd \& Valtonen 1990; Gnedin 2003; Murante et al. 2004; Lin \& Mohr 2004; Willman et al. 2004; Sommer-Larsen 2006; Conrov et al. 2007; Skibba et al. |2007; Purcell et al. 2007, 2008; Yang et al. 2009; Rudick et al. 2009, 2011). This has been studied in great detail at the scale of individual galaxies, often categorized as "stellar halos" or "tidal streams" (Morrison 1993; Sackett et al. 1994; Wetterer \& McGraw 1996; Morrison et al. 1997; Weil et al. 1997; Chiba \& Beers 2000; Ivezić et al. 2000; Lequeux et al. 1998; Abe et al. 1999; Morrison et al. 2000; Yanny et al. 2000; 
Siegel et al. 2002; Irwin et al. 2005; Zibetti \& Ferguson 2004; Guhathakurta et al. 2005; Chapman et al. 2006; Kalirai et al. 2006; McConnachie et al. 2006; Hood et al. 2007; Mackey et al. 2010; Bailin et al. 2011), galaxy groups (Feldmeier et al. 2001; Castro-Rodríguez et al. 2003; White et al. 2003; Da Rocha \& Mendes de Oliveira 2005; Aguerri et al. 2006; Feldmeier 2006; Da Rocha et al. 2008), and galaxy clusters (where it is known as the intra-cluster light, or ICL, e.g., Gallagher \& Ostriker 1972; Merritt 1983; Melnick et al. 1977; Thuan \& Kormendv 1977; Bvrd \& Valtonen 1990; Uson et al. 1991; Bernstein et al. 1995; Calcáneo-Roldán et al. 2000; Gnedin 2003; Murante et al. 2004; Lin \& Mohr 2004; Willman et al. 2004; Mihos et al. 2005; Zibetti et al. 2005; Krick et al. 2006; Sommer-Larsen 2006; Conrov et al. 2007; Seigar et al. 2007; Gonzalez et al. 2007; Pierini et al. 2008; Rudick et al. 2009, 2011; Toledo et al. 2011; Romanowsky et al. 2012).

In this paper, we seek to understand the liberation of stars from satellite galaxies by connecting stellar mass $\operatorname{los} \mathrm{S}^{2}$ to subhalo dark matter mass loss using galaxy clustering and intrahalo light observations. We employ the model for halo substructure introduced in Zentner et al. (2005, hereafter Z05) in order to constrain this relationship. We compare our model predictions to observations of the IHL over a large range of host halo mass scales. The aim of this paper (Paper I) is to introduce our modeling framework and its predictive power. In a forthcoming paper (Paper II) we will extend our analysis to high redshift in order to study the assembly of the IHL across cosmic time.

The paper is laid out as follows. In $\S 2$ we discuss the motivation for this study. In $\S[$ we review the Z05 model for cold dark matter (CDM) substructure. In $\S 4$ we describe our models that connect stellar mass loss to dark matter mass loss and in $\S 5$ we demonstrate how we constrain these models using galaxy clustering. In $\S 6$ we show the luminosity dependence of satellite galaxy stellar mass loss. In $\S 7$ we use our models to make IHL predictions, and compare to observations at low redshift. Finally, in $\S 8$ we give a summary of our results and discuss directions for future work.

\section{MOTIVATION}

A simple, yet remarkably powerful technique for connecting dark matter halo mass to either stellar mass or luminosity has emerged in recent years. By assuming a monotonic relation between halo mass (or maximum circular velocity $\left.V_{\max }=\max [\sqrt{G M(<r) / r}]\right)$ and luminosity $L$ (or stellar mass) one can "abundance match" to make the correspondence between dark matter (sub)halos and an observable galaxy property (e.g., Kravtsov et al. 2004a; Vale \& Ostriker 2004; Tasitsiomi et al. 2004; Vale \& Ostriker 2006; Conrov et al. 2006; Conrov \& Wechsler 2009; Moster et al. 2010; Behroozi et al. 2010; Guo et al. 2010; Simha et al. 2010). For example, this can be done by matching the observed number density of galaxies, $n_{g}$, above some luminosity to the number density of

\footnotetext{
2 By "stellar mass loss", we refer to stars being stripped from a galaxy and not gas lost from stars via winds.
}

halos and subhalos, $n_{h}$, above a certain $V_{\max }$,

$$
n_{g}(>L)=n_{h}\left(>V_{\max }\right) \text {. }
$$

This yields an implicit relationship between $L$ and $V_{\max }$ that preserves the observed luminosity function of galaxies.

Conroy et al. (2006) used this method to assign luminosities to halos and subhalos in a cosmological Nbody simulation at several redshifts. They predicted the luminosity-dependent clustering of galaxies and found that the two-point correlation function $(2 \mathrm{PCF})$ of halos and subhalos matched that of galaxies for a wide range of luminosities and redshifts. The authors made the physically-motivated choice that $V_{\max }$ should be the maximum circular velocity of subhalos at the time of accretion, $V_{\max }^{\text {acc }}$, instead of at the time of observation (see also Nagai \& Kravtsov 2005; Vale \& Ostriker 2006; Berrier et al. 2006).

The reasoning behind this choice is as follows. Upon merging into a larger host, the $V_{\max }$ of a subhalo will decrease due to mass loss (Havashi et al. 2003; Kravtsov et al. 2004a). Dark matter on the periphery of the subhalo will be lost first, because it is less bound to the subhalo. On the other hand, the stellar mass of the satellite galaxy is concentrated at the center of the subhalo and thus more tightly bound. Tidal stripping can significantly alter the surrounding subhalo, but possibly leave the galaxy largely unperturbed for some period of time (using N-body simulations, Peñarrubia et al. 2008a found that halos need to lose $\sim 90 \%$ of their original mass before tides begin to affect the kinematics of stars). Consequently, while $V_{\max }$ of the subhalo decreases, the stellar mass of the galaxy may remain unchanged for long periods of time. It follows that galaxy observables such as luminosity or stellar mass should correlate with $V_{\text {max }}^{\text {acc }}$ instead of the final $V_{\max }$ (Nagai \& Kravtsov 2005). Conroy et al. (2006) lent empirical support to this picture by showing that the choice of $V_{\max }^{\mathrm{acc}}$ was essential in order to achieve agreement with the observed clustering of galaxies.

It is well known that galaxy groups and clusters are replete with diffuse stellar material. This material is widely thought to be the remains of disrupted satellites (see $\S 1$ ). However, the choice to associate galaxy luminosity with $V_{\max }$ at the time of satellite accretion is tantamount to assuming that no stars become unbound from satellite galaxies. In this case, why can using $V_{\max }^{\text {acc }}$ accurately reproduce the observed clustering?

There is a crucial subtlety to simply using $V_{\max }^{\mathrm{acc}}$ for abundance matching. When matching galaxy and subhalo number densities with the $L-V_{\max }$ relation by choosing $V_{\max }^{\mathrm{acc}}$, there is an inherent second threshold in the final $V_{\max }$ of subhalos. This is a threshold below which objects are "operationally removed from consideration" due to the limited resolution of the simulation that is used to perform the calculation. In the study of Conroy et al. (2006), this threshold was $\sim 80 \mathrm{~km} \mathrm{~s}^{-1}$ - the completeness limit of halos in their simulation. Therefore, an accreted subhalo will be artificially destroyed if it becomes sufficiently small such that its structure is not well resolved within the simulation. These so-called "orphans" are neglected and they unwittingly act to model the stripping of stars. The 
galaxies that would have been associated with these halos, had they not become unresolved, are effectively removed from the final galaxy sample, just as if they were disrupted. This elimination of subhalos directly affects the $2 \mathrm{PCF}$, which is very sensitive to subhalo abundance (see Watson et al.|2011). Including these orphans should result in a boost of the small-scale correlation function, implying that pure abundance matching using $V_{\max }^{\text {acc }}$ may not accurately reproduce the observed clustering on small scales (see Kitzbichler \& White 2008; Moster et al. 2010; Wetzel \& White 2010). In fact, we have performed a test in which we selected a $V_{\max }^{\text {acc }}>210 \mathrm{~km} \mathrm{~s}^{-1}$ (corresponding to $\sim L^{*}$ galaxies and brighter) threshold with two, secondary final $V_{\max }$ thresholds, $V_{\max }>20 \mathrm{~km} \mathrm{~s}^{-1}$ and $V_{\max }>80 \mathrm{~km} \mathrm{~s}^{-1}$, to mimic this resolution limit effect. Increasing this threshold from 20 to $80 \mathrm{~km} \mathrm{~s}^{-1}$ resulted in a $\sim 20 \%$ decrease in the $2 \mathrm{PCF}^{3}$ at scales less than $1 \mathrm{Mpc}$. We speculate that below this resolution limit is where much of the stripped stellar material may originate.

The above discussions bring us to the motivation of this work. Including stellar mass loss in the abundance matching technique is necessary to describe the observed clustering of galaxies in a manner that does not include implicit selections. Observed galaxy clustering therefore has the potential to constrain the typical amount of stellar mass loss from a subhalo. In this work, we develop models that relate satellite galaxy stellar mass loss to subhalo dark matter mass loss. We use these models, together with the abundance matching technique, to predict the observed $2 \mathrm{PCF}$ (see $\S 4$ and $\S[5$ for details). Comparing to measurements allows us to constrain our stellar mass loss models. This requires a detailed understanding of the evolution of subhalos within hosts. We use the Z05 analytic model for halo substructure, which is not subject to any intrinsic resolution effects. The model is capable of tracking surviving subhalos down to $V_{\text {max }} \ll 80 \mathrm{~km} \mathrm{~s}^{-1}$, so predictions are not affected by the "orphan" population of galaxies. Our models yield stellar mass loss histories from satellite galaxies, so we can make predictions for the amount of intrahalo light at varying scales and compare to observations. This provides an independent check of our stellar mass loss constraints. This modeling can also be done at varying redshifts in order to study the evolution of stellar mass loss and the assembly of IHL over time. Ultimately, this investigation could enable a single model to track the stellar-to-halo mass relation as a function of time such that abundance matching at multiple redshifts would be unnecessary.

\section{THE SUBHALO EVOLUTION MODEL}

We construct models in which the stellar mass loss of a satellite galaxy is linked to the dark matter mass loss of its subhalo. Consequently, we require a detailed understanding of the evolution of subhalos within hosts. For this we use the Z05 model, which is based on Zentner \& Bullock (2003) and is similar to the independent models of Tavlor \& Babul (2004, 2005a,b) and Peñarrubia \& Benson (2005), and shares many features with other approximate treatments of subhalo populations (Oguri \& Lee 2004; van den Bosch et al.

3 We explain the tools we use to calculate correlation functions in $\S 5$
2005; Faltenbacher \& Mathews 2005; Purcell et al.|2007; Giocoli et al. 2008, 2009; Gan et al. 2010; Yang et al. 2011b). The Z05 model produces subhalo mass functions, occupation statistics, and radial distributions within hosts that are in good agreement with a number of highresolution $N$-body simulations (Z05, and the recent comparison in Koushiappas et al. 2010).

The analytic model proceeds as follows. For a host halo at a given redshift $z$, and mass $M$, we generate a halo merger tree using the mass-conserving implementation of the excursion set formalism (Bond et al. 1991; Lacey \& Cole 1993, 1994; see Zentner 2007 for a review) developed by Somerville \& Kolatt (1999). This provides the entire history of all halos that merged to form the final, host halo. We assign all halos Navarro et al. (1997, hereafter NFW) density profiles with concentrations determined by their merger histories according to Wechsler et al. (2002). When a halo merges into a larger halo, it becomes a subhalo and is assigned initial orbital parameters drawn from distributions measured in N-body simulations (Z05; also see Benson 2005 for similar formalisms). We then integrate each subhalo orbit within the gravitational field of the host, subjecting the subhalos to orbital decay via dynamical friction and mass loss through tides and interactions. We estimate dynamical friction with an updated form of the Chandrasekhar (1943) approximation (Hashimoto et al. 2003; Zentner \& Bullock 2003), and allow for dark matter mass loss beyond the tidal radius on a timescale comparable to the local dynamical time. Finally, we account for internal heating so that scaling relations describing the internal structures of subhalos are obeyed (Havashi et al. 2003; Kazantzidis et al. 2004; Kravtsov et al. 2004b). We refer the reader to Z05 for specific details of these ingredients.

The lack of a resolution limit in the Z05 model allows us to track subhalos regardless of how much mass they have lost. Therefore, we do not lose subhalos due to mass loss. However, we do consider a subhalo to be effectively "destroyed" if its orbital apocenter sinks to less than $r_{\text {apo }}<5 \mathrm{kpc}$ distance from the center of its host halo. This choice is physically motivated because the galaxy within such a subhalo would likely have merged with the central galaxy, or at least be observationally indistinguishable from it. This criterion thus models the cannibalism of satellite galaxies by central galaxies. We show in $\S 7$ that inclusion of these cannibalized subhalos in our predictions for intrahalo light is negligible, so this criterion actually has little effect on our modeling results. In practice, subhalos lose the vast majority of their mass prior to achieving such small apocenters, so this cut serves only to terminate the integration of that particular orbit. In the end, we amass catalogs of all surviving subhalos in the final host halo at the time of "observation" and we know exactly how much dark matter has been lost from each subhalo. A halo that merges into a larger host may contain subhalos of its own. These "subs-of-subs" of sufficiently high mass to host an observable galaxy are only abundant inside very large host masses. They are present in our model, but rare.

To properly sample the distribution of halos in the universe, we compute subhalo populations for a grid of host halo masses in the range $11 \leq \log \left(M / h^{-1} M_{\odot}\right) \leq 15$ (in steps of 0.1$)$. To account for statistical variation among 

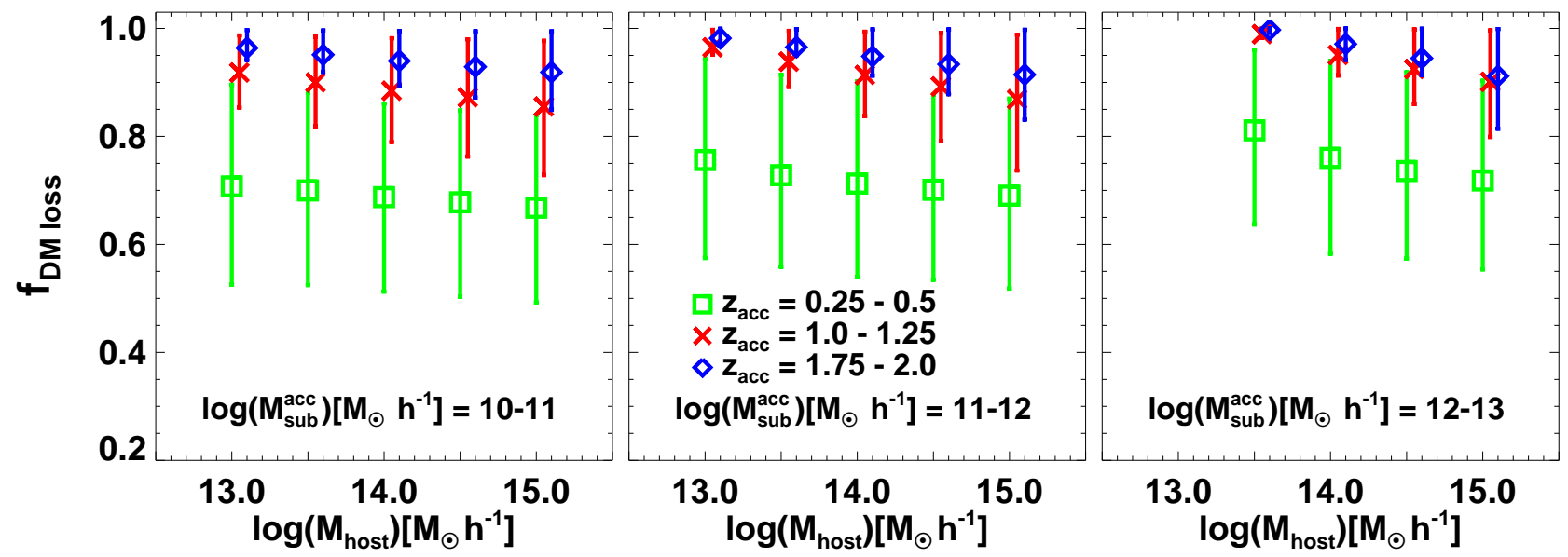

Figure 1. The average fraction of dark matter lost from subhalos, $f_{\mathrm{DM} \text { loss }}=\left(M_{\mathrm{acc}}^{\mathrm{dm}}-M_{\mathrm{fin}}^{\mathrm{dm}}\right) / M_{\mathrm{acc}}^{\mathrm{dm}}$, as a function of host halo mass for three bins of subhalo accretion epoch $z_{\text {acc }}$ (shown by the three point types), and three bins of subhalo mass (at accretion; shown by the three panels), according to the Z05 semi-analytic model. Each point represents an average over all subhalos in a given bin of accretion epoch and subhalo mass, from 500 model realizations of a specific host mass, and errorbars show the $1 \sigma$ scatter. The points representing different accretion epoch bins are slightly staggered for clarity. Accretion time is the dominant factor that determines $f_{\mathrm{DM}}$ loss, with subhalos that have merged earlier having more time to be stripped of their dark matter.

halos and subhalos, we perform 500 statistical realizations of the subhalo population at each host mass. The result is 500 host halos along with their subhalos at each of 41 distinct masses, giving a total of 20,500 distinct subhalo populations. The model predicts the amount of dark matter lost from each subhalo as it orbits in the tidal field of its host halo. Figure 1 shows the fraction of subhalo mass lost as a function of host halo mass, in bins of accretion epoch $z_{\text {acc }}$, and subhalo mass (at accretion). This fraction is defined as $f_{\mathrm{DM} \text { loss }}=\left(M_{\mathrm{acc}}^{\mathrm{dm}}-M_{\mathrm{fin}}^{\mathrm{dm}}\right) / M_{\mathrm{acc}}^{\mathrm{dm}}$, where $M_{\mathrm{acc}}^{\mathrm{dm}}$ is the subhalo mass at accretion and $M_{\mathrm{fin}}^{\mathrm{dm}}$ is the final subhalo mass. Each panel of the figure represents a bin of subhalo mass and the three sets of points in each panel represent bins of accretion epoch: $z_{\text {acc }}=0.25-0.5$ (green circles), $z_{\text {acc }}=1.0-1.25$ (red $X$ symbols), and $z_{\text {acc }}=1.75-2.0$ (blue diamonds). Each point thus represents an average over all subhalos in a bin of accretion epoch and subhalo mass, from the 500 model realizations of a specific host halo mass, and errorbars show the $1 \sigma$ scatter. It is clear that accretion time is crucial towards determining $f_{\mathrm{DM}}$ loss, with subhalos that have merged earlier having more time to be stripped of their dark matter. Moreover, the scatter in $f_{\mathrm{DM}}$ loss shrinks for earlier accretion times. At fixed accretion epoch, the average value of $f_{\mathrm{DM}}$ loss is remarkably constant. However, there is a slight increase as we move to lower host halo mass at fixed subhalo mass, or as we move to higher subhalo mass at fixed host mass. This is a result of dynamical friction playing a stronger role for subhalos that are considerable in size compared to their host halos. The larger a subhalo relative to its host, the more rapidly its orbit will decay and it will sink within the host potential. The tidal field of the host is stronger towards the host center, and this induces more dark matter mass loss.
We now present the models we use to describe satellite galaxy stellar mass loss. The exact way in which stars are stripped from the subhalo they inhabit is likely quite complicated. However, we can make some simple, physically-motivated approximations aimed at capturing the gross, relevant behavior. In this sense, we pursue the question of the evolution of stellar mass using a philosophy similar to that which underlies abundance matching. We aim to make a set of minimal, yet effective assumptions that serve to distill the enormous amount of information contained in survey data. Indeed, we aim in part to extend the abundance matching techniques by making the lower threshold for stellar mass explicit, rather than implicit.

We consider two models in which we relate the amount of satellite galaxy stellar mass lost to the corresponding amount of subhalo dark matter lost. Combined with the Z05 model that makes detailed predictions for dark matter mass loss, these models can predict the stellar mass loss for any given halo. It is important to note that the Z05 prescription for mass loss does not include the effects that baryons can have on dark matter. Adiabatic Contraction (AC) (Blumenthal et al. 1986; Ryden \& Gunn 1987; Gnedin et al. 2004) may increase the central density as the gas condenses and sinks to to the center of the dark matter potential well (Diemand et al. 2004; Fukushige et al. 2004; Reed et al. 2005; Del Popolo \& Kroupa 2009). Conversely, processes during halo formation, such as gravitational heating from merger events, can counteract AC (e.g., Zappacosta et al. 2006) and, in fact, the central density may decrease through baryonic feedback (Governato et al. 2012). These effects can impact the survival of satellite galaxies (see Peñarrubia et al. 2010), however consideration of these detailed effects in our modeling is beyond the objective of this paper. 

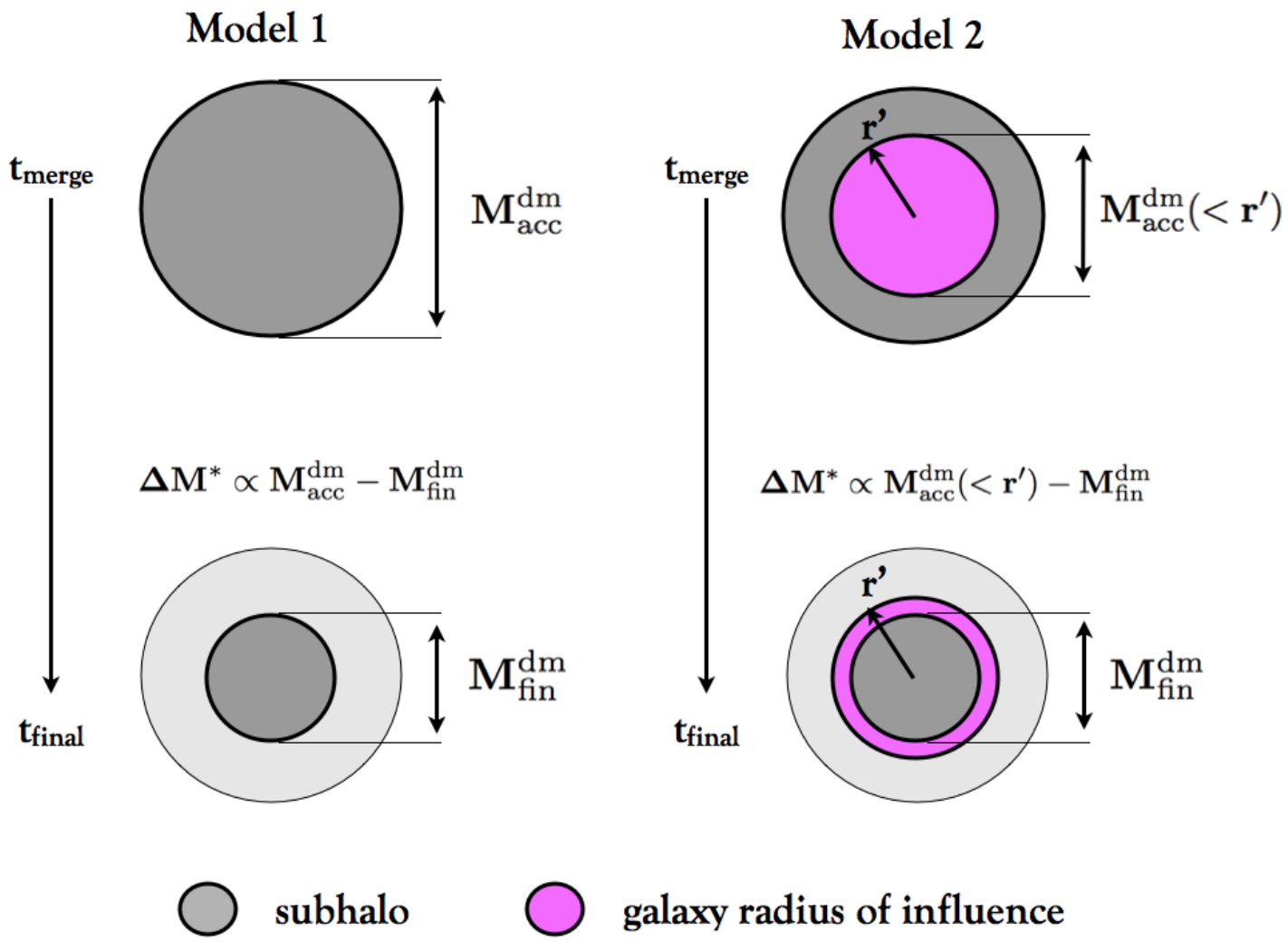

galaxy radius of influence

Figure 2. Schematic of how satellite galaxy stellar mass loss occurs in Model 1 and Model 2. From the time the subhalo merges into the host halo, $t_{\text {merge }}$, until the final redshift under consideration, $t_{\text {final }}$, our semi-analytic subhalo model predicts the amount of dark matter mass loss, $\Delta M^{\mathrm{dm}} \equiv M_{\mathrm{acc}}^{\mathrm{dm}}-M_{\mathrm{fin}}^{\mathrm{dm}}$. Model 1 assumes that the amount of stellar mass lost, $\Delta M^{*}$, is proportional to $\Delta M^{\mathrm{dm}}$. Model 2 defines a radius, $r^{\prime}$, such that stellar mass is only lost if dark matter is lost within that radius. If this is the case, then $\Delta M^{*}$ is only proportional to the amount of dark matter lost inside of this radius. See $\S$ 的or detailed descriptions of the models.

Our first model sets the fraction of stellar mass that is lost from a galaxy to a fixed proportion of the fraction of dark matter that is lost from its subhalo. The model works as follows. Any halo of sufficiently large dark matter mass will have some stellar mass associated with the galaxy it contains at the time of accretion, $M_{\text {acc }}^{*}$. This stellar mass will be some fraction of the mass in dark matter, $M_{\mathrm{acc}}^{\mathrm{dm}}$. After the halo merges into a larger halo, becoming a subhalo, it orbits within the host halo potential and loses mass. At the time of observation, the subhalo has a smaller mass, $M_{\text {fin }}^{\mathrm{dm}}$. We relate the fraction of stellar mass that is lost during this time to the fraction of dark matter mass that is lost through a single parameter $\epsilon$,

$$
\begin{gathered}
\frac{\Delta M^{*}}{M^{*}}=\epsilon \frac{\Delta M^{\mathrm{dm}}}{M^{\mathrm{dm}}}, \\
\frac{M_{\mathrm{acc}}^{*}-M_{\mathrm{fin}}^{*}}{M_{\mathrm{acc}}^{*}}=\epsilon \frac{M_{\mathrm{acc}}^{\mathrm{dm}}-M_{\mathrm{fin}}^{\mathrm{dm}}}{M_{\mathrm{acc}}^{\mathrm{dm}}},
\end{gathered}
$$

which can be re-written as,

$$
M_{\mathrm{fin}}^{*}=M_{\mathrm{acc}}^{*}\left[1-\epsilon \times\left(\frac{M_{\mathrm{acc}}^{\mathrm{dm}}-M_{\mathrm{fin}}^{\mathrm{dm}}}{M_{\mathrm{acc}}^{\mathrm{dm}}}\right)\right]
$$

The left-hand side of Figure 2 is a cartoon schematic of how stellar mass loss occurs in Model 1. From the time the subhalo merges into the host halo, $t_{\text {merge }}$, until the final redshift under consideration, $t_{\text {final }}$, the Z05 model (see $\S 3$ for model details) predicts the amount of subhalo dark matter mass loss $\Delta M^{\mathrm{dm}}$ with the stellar mass loss $\Delta M^{*}$ being related to the dark matter mass loss via $\epsilon$. Therefore, we are left with a simple parametric equation governed by a single free parameter. For example, if $\epsilon=0.5$, then a subhalo that loses $50 \%$ of its dark matter will lose $25 \%$ of its stellar mass. As we mentioned above, stellar mass loss should be less efficient than dark matter mass loss, so we should generally expect $\epsilon<1$.

In order to compute the final stellar mass of a galaxy via Eqn. 4] we need to know the stellar mass of a satellite galaxy as a function of the subhalo mass at the time of accretion. The stellar mass of a galaxy depends on many physical processes, including mergers, gas cooling, star formation, feedback from supernovae, feedback from active galactic nuclei, making ab initio predictions highly non-trivial. However, there are several empirical methods for obtaining the stellar-to-halo mass relation (SHMR), which have recently appeared in the literature (e.g., Yang et al. 2003; Wang et al. 2006; Conrov \& Wechsler 2009; Wang \& Jing 2010; Moster et al.|2010; Guo et al.|2010; Behroozi et al. 2010; Neistein et al. 2011). We assign stellar masses to halos with the SHMR of Behroozi et al. (2010, hereafter 


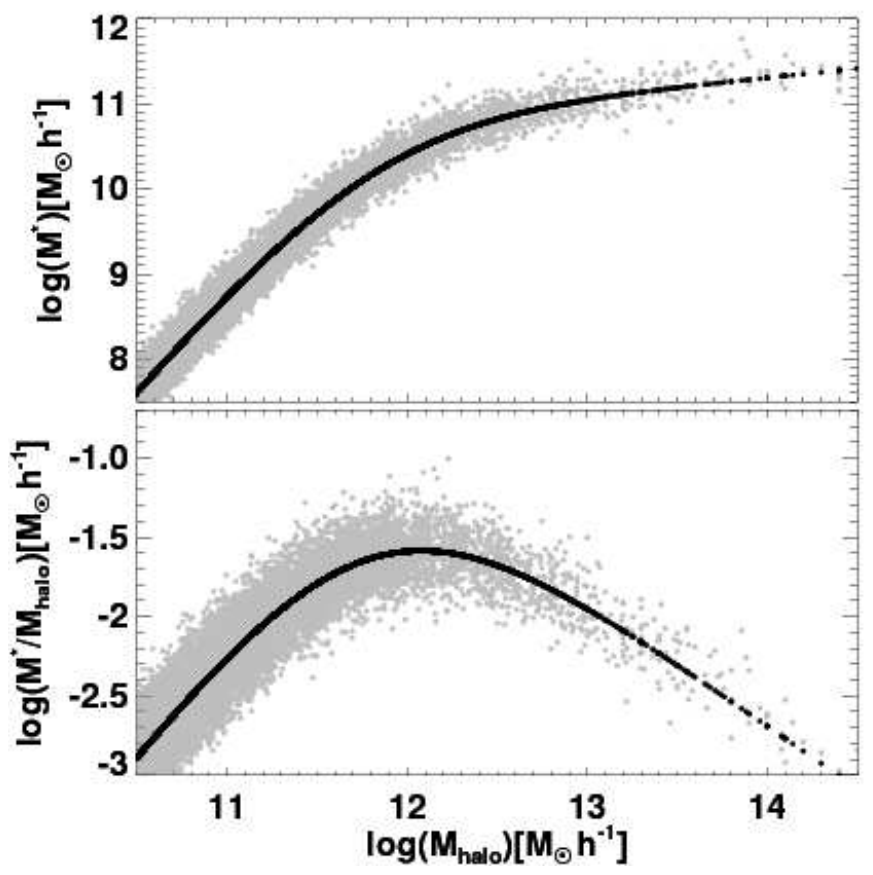

Figure 3. The stellar-to-halo mass relation (SHMR) for our host halos and subhalos with the adopted Behroozi et al. (2010) SHMR. The black curve shows the mean of the SHMR and grey points illustrate the assumed 0.15 dex scatter by sampling 1 million halos and subhalos from all of the model realizations. The bottom panel is the same as the top with the y-axis divided by halo mass to highlight the characteristic mass $\left(\sim 10^{12} h^{-1} M_{\odot}\right)$ where star formation is most efficient.

B10). Specifically, we employ the relation used in Eqn. 21 of B10, with the mean parameter values given by the $\mu=\kappa=0$ model in their Table 2. Therefore, for every halo and subhalo in our catalog, we can use the halo mass at the time of accretion to assign a stellar mass to the galaxy hosted by the halo. The top panel of Figure 3 shows how stellar masses are related to our host halos and subhalos with the B10 SHMR. The black curve shows the mean of the SHMR and grey points illustrate the assumed 0.15 dex scatter given in B10. We show one million points randomly drawn from the full distribution of halos and subhalos over the host halo mass range $11 \leq \log \left(M / h^{-1} M_{\odot}\right) \leq 15$. Stellar mass rapidly increases as a function of halo mass at low masses before turning over and becoming shallower at higher host masses. The bottom panel is the same as the top with the y-axis divided by halo mass in order to highlight the characteristic mass where this turnover occurs, $M_{\text {halo }} \sim 10^{12} h^{-1} M_{\odot}$. This characteristic mass is the halo mass at which star formation is most efficient.

For most applications, SHMRs are developed by linking the halo mass function to the galaxy stellar mass function (SMF) through abundance matching. This is typically done using the subhalo mass at accretion which, by definition, presumes that no stellar stripping occurs. However, even if stellar stripping occurs (i.e., if $\epsilon$ is nonzero) we can still use a SHMR relation that assigns stellar mass at the time the subhalo merges, due to the fact that the SMF is strongly dominated by central galaxies. We emphasize that while there are many SHMRs in the literature, we are not very sensitive to the partic- ular choice of SHMR. As we will discuss in $\S$ 5, we assign stellar masses to halos and subhalos and then rank them in stellar mass in order to find the stellar mass cutoff $\left(M_{\text {fin }}^{*}\right)$ that matches the observed number density of a given galaxy luminosity threshold sample. Therefore, two SHMRs that yield the same rank order for the halos and subhalos will be indistinguishable from each other, even though the values of $M_{\text {fin }}^{*}$ will be different (as well as the mass-to-light ratios). All published SHMRs are monotonically increasing functions, so this behavior is general. On the other hand, the scatter in the SHMR is important, as this will change the rank order. We use the B10 scatter of 0.15 dex throughout our analysis, though we test the effect of changing the scatter on our results in $\S 5.1$. We also note that we do not consider the fact that the stellar mass of a galaxy may actually increase for some time after merging and becoming a satellite, as it has been recently shown that star formation in active satellites may continue for several Gyr (see Wetzel et al. 2011). Such detailed modeling is beyond the scope and intention of this work.

In summary, we take the following steps in Model 1 for assigning final stellar masses to an ensemble of halos.

1. We use the Z05 model for subhalo evolution to obtain a list of halos and subhalos for a range of host halo masses, and we determine the fractional amount of dark matter mass lost from each subhalo.

2. We use the B10 stellar-to-halo mass relation to assign stellar masses to host halos and subhalos at the time of accretion.

3. For every subhalo, we compute a final stellar mass by relating the fractional amount of stellar mass lost to the fractional amount of dark matter mass lost through the single free parameter $\epsilon$, as described in Eqn. 4.

\subsection{Model 2}

Model 1 operates under the basic assumption that the fractional amount of satellite galaxy stellar mass loss is proportional to subhalo dark matter loss through the free parameter $\epsilon$. This means that even a small amount of dark matter stripping will be accompanied by some stellar stripping. However, as we argued in $\S 2$, a subhalo may lose a considerable amount of dark matter before its galaxy is significantly disturbed. For example, subhalos in high-resolution simulations lose a significant fraction of mass at their outskirts rapidly upon merging into a larger system, while their interiors remain unaltered by this mass loss (e.g., Diemand et al. 2007). Accordingly, we consider an alternative model that incorporates a delay between the initial loss of dark matter mass and stellar mass loss.

Our second model states that stellar mass will only be lost if a sufficient amount of dark matter is lost first. The model works as follows. We first define an approximate radius of influence that a galaxy has within its subhalo, corresponding to the region within which the gravity due to the stellar component is comparable to that from dark matter. To estimate this radius, we first assign a stellar mass to each subhalo at the time of accretion, $M_{\text {acc }}^{*}$, using 
the B10 formalism. Next we assume, for simplicity, that this is a point mass and we do not consider the total amount of "cold baryons" or assume a galaxy profile. To find the radius of influence, $r_{\mathrm{infl}}$, of the stellar mass associated with the galaxy, we calculate where the dark matter mass enclosed within the subhalo is equal to $M_{\mathrm{acc}}^{*}$. We thus integrate the NFW mass profile,

$$
M_{\mathrm{acc}}^{\mathrm{dm}}\left(<r_{\mathrm{infl}}\right)=4 \pi \rho_{0} r_{s}^{3}\left[\ln \left(1+\frac{r_{\mathrm{inf}}}{r_{s}}\right)-\frac{r_{\mathrm{infl}} / r_{s}}{1+r_{\mathrm{infl}} / r_{s}}\right],
$$

where $\rho_{0}$ and $r_{s}$ are the NFW parameters, and solve for $r_{\text {inf }}$ by setting $M_{\mathrm{acc}}^{\mathrm{dm}}\left(<r_{\mathrm{inf}}\right)=M_{\mathrm{acc}}^{*}$.

The radius $r_{\text {infl }}$ gives the rough scale within which mass must be lost before significant stellar mass is lost; however, we allow for flexibility in this final prescription for stellar mass loss. We assume that a galaxy will start losing stellar mass once dark matter is stripped from inside a radius that scales linearly with $r_{\text {infl }}$. We define a new radius, $r^{\prime}=\psi \times r_{\text {infl }}$, where $\psi$ is a free parameter of order unity. If the subhalo loses so much dark matter that its final mass is less than $M_{\mathrm{acc}}^{\mathrm{dm}}\left(<r^{\prime}\right)$, we allow stellar stripping to occur. Furthermore, we assume that the fraction of stellar mass lost is equal to the fraction of dark matter lost after the bound mass of the subhalo crosses below this threshold. The final stellar mass of the galaxy is thus

$$
M_{\mathrm{fin}}^{*}=M_{\mathrm{acc}}^{*}\left[1-\max \left\{0,\left(\frac{M_{\mathrm{acc}}^{\mathrm{dm}}\left(<r^{\prime}\right)-M_{\mathrm{fin}}^{\mathrm{dm}}}{M_{\mathrm{acc}}^{\mathrm{dm}}\left(<r^{\prime}\right)}\right)\right\},\right.
$$

which is analogous to Eqn. 4.

The right-hand side of Figure2 2 illustrates how Model 2 works. After accretion, if the subhalo shrinks enough due to mass loss such that its mass at the final redshift of interest is less than $M_{\mathrm{acc}}^{\mathrm{dm}}\left(<r^{\prime}\right)$, then stellar mass will be lost $\left(\Delta M^{*}\right)$ in proportion to the additional amount of dark matter lost. However, if mass loss does not reduce the subhalo mass to less than $M_{\mathrm{acc}}^{\mathrm{dm}}\left(<r^{\prime}\right)$, no stellar mass is lost. Model 2 incorporates a delay between the stripping of the outer layers of dark matter and the stripping of the stellar material residing in the depths of the subhalo potential well. The single free parameter of Model 2, $\psi$, allows us to vary the amount of lag. As was the case for $\epsilon=0$ in Model 1, setting $\psi=0$ in Model 2 leads to zero stellar mass loss in all subhalos.

In summary, we take the following steps in Model 2 for assigning final stellar masses to an ensemble of halos.

1. We obtain a population of halos and assign stellar masses at the time of accretion as described in the first two steps of the Model 1 summary.

2. For each subhalo, we calculate $r_{\text {inf }}$ using Eqn. 5 We then scale this radius using the free parameter $\psi$, to get $r^{\prime}=\psi \times r_{\text {infl }}$.

3. We calculate the dark matter mass at accretion that is enclosed by $r^{\prime}, M_{\mathrm{acc}}^{\mathrm{dm}}\left(<r^{\prime}\right)$. If insufficient dark matter loss has occurred, such that the final subhalo mass is greater than this, then we assume that no stellar mass loss has taken place. Otherwise, we estimate a final stellar mass using Eqn. 6.
It is important to note the effect of allowing the stellar and dark matter components to have distinct density profiles. Using N-body simulations, Peñarrubia et al. $(2008 \mathrm{~b})$ studied the tidal evolution of satellite galaxies embedded in dark matter halos. They adopted the observationally-motivated assumption that stellar components have cored (King) profiles, while dark matter follow cuspy NFW models. They helped explain the extremely large mass-to-light (M/L) ratios of ultrafaint MW dwarfs by showing that the cuspy nature of the NFW profile keeps the central dark matter component more tightly bound than the stars. For our Model 2, we have taken $\epsilon=1$ for simplicity in order to illustrate the basic features of a simple model as well as the manner in which it can be constrained. Taking $\epsilon=1$ in this model allows for stellar mass loss with a particular time dependence. Specifically, M/L ratios can evolve only when mass loss does not occur interior to the threshold radius $r^{\prime}$. Any additional mass loss interior to $r^{\prime}$ keeps the $\mathrm{M} / \mathrm{L}$ ratios fixed. There are two considerations to this end. First, allowing $\epsilon$ to take on a value other than 1 would give a time-dependent $\mathrm{M} / \mathrm{L}$ ratio even for mass loss interior to $r^{\prime}$. However, our goal is to present a simple model and illustrate constraints on such a model. A second parameter would lead to inevitable degeneracies among parameters and introduce complexities that would detract from the broader point of the current paper, and we show below that a single-parameter model adequately describes various observational data. Second, in even greater detail, we could consider a time-varying $\epsilon=\epsilon(t)$, to describe a very broad range of variations in $\mathrm{M} / \mathrm{L}$ ratios with time. Though not currently necessary to describe the data we consider, such a model may be more physically appropriate, and the time variation of $\epsilon$ could be derived, at least approximately, from specific assumed stellar mass profiles. In follow-up work, we are building on the complexity of the models we present here, though the details of these broader models are well beyond the scope and intent of this paper.

\section{CONSTRAINING SATELLITE GALAXY STELLAR MASS LOSS USING GALAXY CLUSTERING}

We now turn to constraints on the relationship between stellar mass and halo mass imposed by the clustering of galaxies. Our two stellar mass loss models specify the final stellar mass in any halo or subhalo, given a value for the parameter $\epsilon$ (for Model 1) or $\psi$ (for Model 2). With stellar masses assigned to all halos and subhalos, we can predict a halo occupation distribution (HOD: e.g., Peacock \& Smith 2000; Scoccimarro et al. 2001; Berlind \& Weinberg 2002; Cooray \& Sheth 2002) for any given stellar mass threshold. In particular, we compute the mean number of galaxies as a function of host halo mass, $\langle N\rangle_{\mathrm{M}}$. We calculate the mean over the 500 host halo realizations at each host mass. We use this function to compute the number density of galaxies by weighting the host halo abundances by $\langle N\rangle_{\mathrm{M}}$ and integrating over all halo masses,

$$
\bar{n}_{g}=\int_{0}^{\infty} d M \frac{d n}{d M}\langle N\rangle_{M} .
$$

We adopt the Warren et al. (2006) halo mass function, $d n / d M$, in this calculation, though our results are not sensitive to the specific choice of mass function. In this 
way, we find the stellar mass threshold that yields a galaxy number density equal to that of the observed sample with which we aim to compare.

We compare our model predictions to four of the Sloan Digital Sky Survey (SDSS: York et al. 2000) luminosity threshold galaxy samples measured by Zehavi et al. (2011). Specifically, we consider volume-limited samples with $r$-band absolute magnitude thresholds of $M_{r} \leq$ $-18,-19,-20$, and -21 (number densities for these samples are listed in Table 2 of Zehavi et al. 2011). We do not consider the fact that the observed relationship between stellar mass and luminosity is not one-to-one (i.e., galaxies of a fixed luminosity can have different stellar masses which, for instance, is manifested as scatter in the Tully-Fisher relation - Bell \& de Jong 2001). However, including this scatter should be similar to increasing the scatter in the B10 relation between dark matter mass and $M_{\text {acc }}^{*}$, which has a very small effect, as we show in $\S 5.1$.

Once we have determined the appropriate stellar mass threshold, we use the resulting HOD to calculate the predicted clustering of the model. We place galaxies within the host halos in a N-body simulation to measure the $2 \mathrm{PCF}$. We use a single realization of the "Consuelo" simulation (with a box size of $420 h^{-1} \mathrm{Mpc}$ and $1400^{3}$ particles), which is part of the LasDamas suite of simulations (McBride et al., in prep.). The cosmology assumed in our semi-analytic subhalo model is set to match that of the LasDamas simulation 4 and is similar to the recent WMAP7 values (Komatsu et al. 2011). We use the spherical over-density (SO) halo finder "Rockstar" (Behroozi et al. 2011) to identify halos. We use an $\mathrm{SO}$ halo finder because the SO algorithm mimics the assumptions made in our semi-analytic model. Both the Z05 model and the halo finder define virial masses based on the virial threshold definition of Bryan \& Norman (1998). We note, however, that our results are not sensitive to the choice of halo finder. We have repeated our analysis using a Friends-of-Friends halo finder and derived similar results. We populate host halos with galaxies according to our $\langle N\rangle_{M}$ model predictions.

In order to compare to data, we convert our realspace correlation functions, $\xi(r)$, to projected correlation functions, $w_{\mathrm{p}}\left(r_{\mathrm{p}}\right)$, by integrating along lines of sight (Davis \& Peebles 1983; Zehavi et al. 2004):

$$
w_{\mathrm{p}}\left(r_{\mathrm{p}}\right)=2 \int_{0}^{y_{\max }} \xi\left(\sqrt{r_{\mathrm{p}}^{2}+y^{2}}\right) d y .
$$

For each luminosity threshold, we integrate out to the same $y_{\max }$ as Zehavi et al. (2011, listed as $\pi_{\max }$ in their Table 2). We predict $w_{\mathrm{p}}\left(r_{\mathrm{p}}\right)$ for the four luminosity samples, given any value of $\epsilon$ from Model 1 or $\psi$ from Model 2 . In order to constrain our stellar mass loss models, we run a grid of $\epsilon$ and $\psi$ values in steps of $\Delta \epsilon=0.1$ and $\Delta \psi=0.05$. For each parameter value and each luminosity threshold, we compute $\chi^{2}$ by comparing our model prediction to the Zehavi et al. (2011) measurement of $w_{\mathrm{p}}\left(r_{\mathrm{p}}\right)$, using only diagonal errors (not the full covariance matrices).

4 Throughout the paper, we work within the standard, vacuumdominated, cold dark matter $(\Lambda \mathrm{CDM})$ cosmological model with $\Omega_{\mathrm{m}}=0.25, \Omega_{\Lambda}=0.75, \Omega_{\mathrm{b}}=0.04, h_{0}=0.7, \sigma_{8}=0.8$, and $n_{\mathrm{s}}=1.0$.

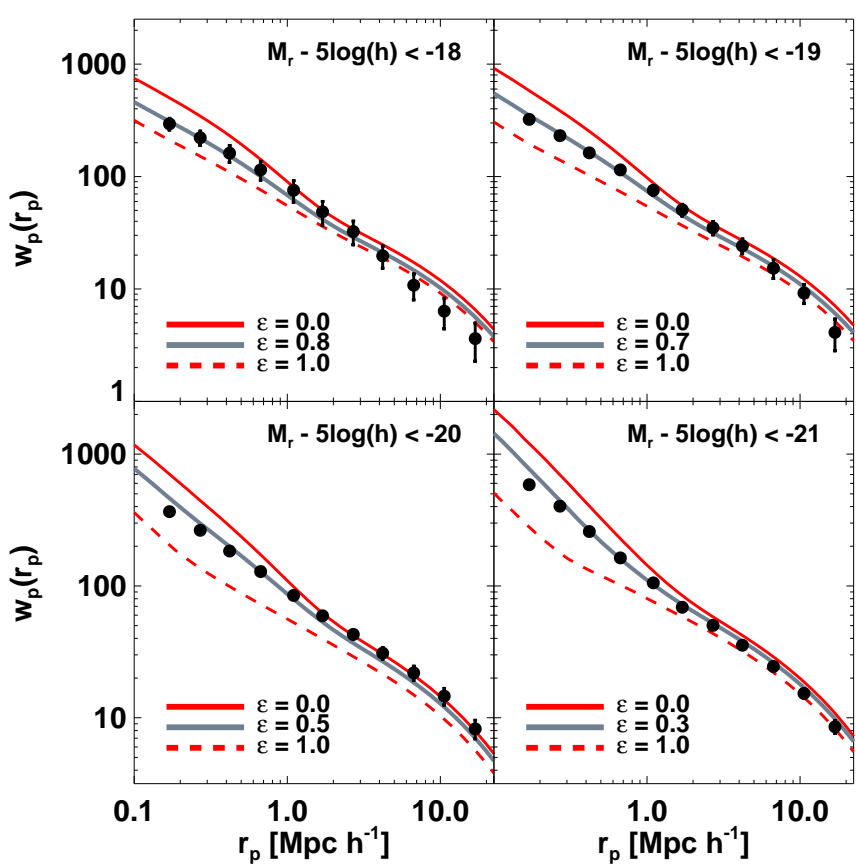

Figure 4. Clustering predictions for Model 1. Model 1 sets the fractional amount of satellite galaxy stellar mass loss to be proportional to the fractional amount of subhalo dark matter mass loss through the free parameter $\epsilon$ (see 4.1 for model details and Fig. 2 for an illustration). Each panel shows results for a different galaxy luminosity threshold. Solid red curves show model predictions for $\epsilon=0$, equivalent to no stellar mass loss. Dashed red curves show the $\epsilon=1$ case, where the stellar mass loss occurs at the same rate as subhalo dark matter mass loss. Grey curves show the $\epsilon$ values that match the observed SDSS clustering measurements of Zehavi et al. (2011, black points).

Figure 4 shows clustering results for Model 1 compared to measurements from Zehavi et al. (2011) for our four SDSS luminosity threshold samples: $M_{r}<$ $-18,-19,-20$ and -21 . To reiterate, Model 1 assumes that the fractional amount of satellite galaxy stellar mass loss is proportional to the fractional amount of subhalo dark matter mass loss with proportionality constant $\epsilon$. Adopting $\epsilon=0$ specifies no stellar mass loss and is equivalent to using $V_{\max }$ at the epoch of accretion for abundance matching. On the other hand, adopting $\epsilon=1$ means that stellar mass is lost at the same rate as dark matter. For each luminosity sample, we show the $\epsilon=0$ and 1 cases as red solid and dashed lines, respectively.

At each luminosity, $\epsilon=0$ predicts small-scale clustering that is stronger than the data indicate and this gets to the essence of using clustering to constrain stellar mass loss. This over-prediction can be attributed to the fact that no stellar mass loss has occurred for satellite galaxies, resulting in too many satellites with high stellar masses. This leads to enhanced clustering on small scales (e.g. Watson et al. 2011). To be consistent with the data, some stellar mass loss needs to occur. However, the $\epsilon=1$ result demonstrates that too much stellar mass loss leads to weaker clustering than the data require. The grey curves in Figure 4 correspond to intermediate amounts of stellar mass loss and represent the $\epsilon$ with the lowest $\chi^{2}$ values 5 Despite the fact that Model 1

${ }^{5}$ We note that none of our model predictions match the large- 


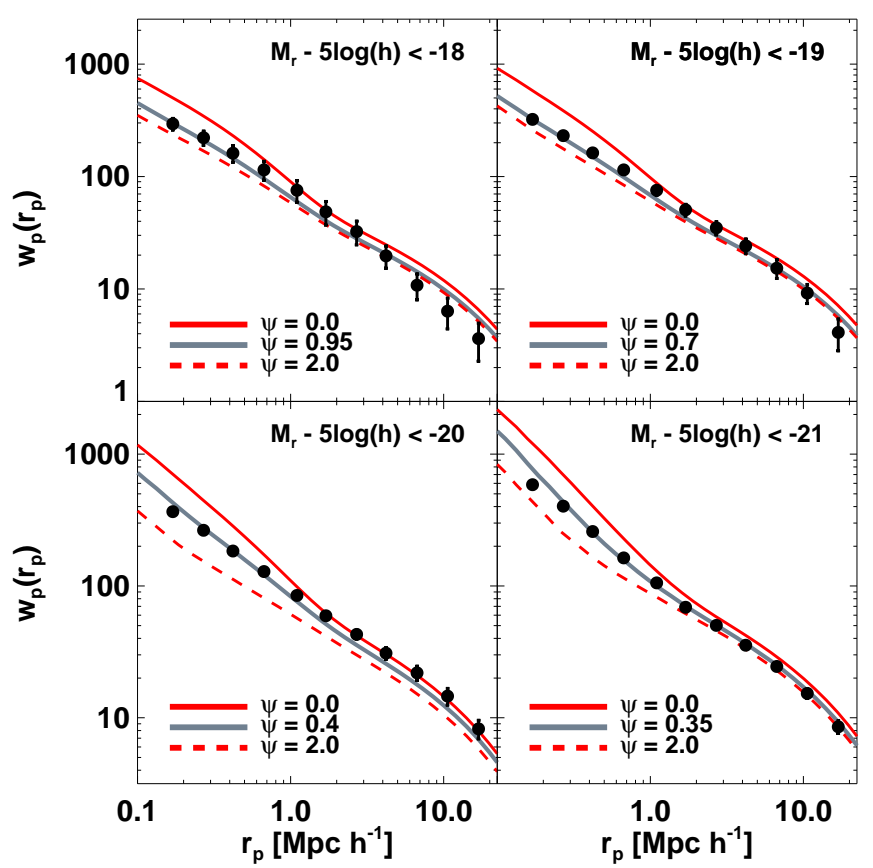

Figure 5. Clustering results for Model 2. Model 2 defines a radius of influence, $r_{\text {infl }}$, for a galaxy within a subhalo. If a subhalo loses sufficient dark matter mass such that it starts to lose mass inside of $r_{\mathrm{inf}}$, then stellar mass is lost at a rate proportional to the subsequent subhalo mass loss. The free parameter $\psi$ allows $r_{\text {inf }}$ to grow or shrink, thus a larger value of $\psi$ means that more stellar mass loss will occur on average for satellite galaxies. Solid red curves show model predictions for $\psi=0$, equivalent to no stellar mass loss, thus the same as $\epsilon=0$ for Model 1. Dashed red curves show the predictions for $\psi=2$, an arbitrary value chosen to represent how too much stellar mass loss will under-predict $w_{\mathrm{p}}\left(r_{\mathrm{p}}\right)$. Grey curves show the $\psi$ values that match the observed clustering for the same luminosity threshold samples as in Fig. 4

paints a simplified picture of how stars are stripped from the galaxies they reside in, we show that it is very effective at matching the observed clustering. Moreover, it is striking that $\epsilon$ decreases with increasing luminosity. We will discuss this luminosity dependence in detail in $\S 6$.

Figure 5] shows similar results for Model 2. This model was designed to allow dark matter on the periphery of a subhalo to be lost due to the strong tidal field of the host, without significantly altering the luminous galaxy residing deep in the core of the subhalo. Model 2 mimics this "lag" by defining a critical radius within each subhalo and only allowing for stellar mass loss if the subhalo loses mass from within that radius. This radius can be varied through the parameter $\psi$. The limit $\psi=0$ corresponds to no stellar mass loss and is equivalent to abundance matching using $V_{\max }$ at accretion. In Model 1, $\epsilon=1$ is analogous to using the subhalo mass at the final redshift output for abundance matching. In Model 2, this happens when the critical radius is larger than the size of the subhalo at accretion. This occurs at a different value of $\psi$ for each subhalo. Thus, we choose an arbitrary value $\psi=2$ to again illustrate how too much stellar mass loss will under-predict clustering. As was the case for Model 1, we are able to match the observed

scale clustering for the $M_{r}<-18$ sample. This may be attributed to the finite volume of the sample, as discussed in $\S 3.2$ of Zehavi et al. (2011). clustering and we see a strong evolution in $\psi$ as a function of luminosity. This emphasizes what was found for Model 1, that low-luminosity galaxies experience more efficient stellar stripping throughout their evolution in a host halo than luminous galaxies.

\subsection{The Effect of Scatter in the Stellar-to-Halo Mass Relation}

Observations indicate that halos of a given mass can host galaxies with a range of luminosities and stellar masses (e.g., van den Bosch et al. 2007; Zheng et al. 2007; Yang et al. 2008; More et al. 2009). For this reason, it is overly restrictive to assume a one-to-one relation between halo mass and stellar mass, whereas a relationship with some intrinsic scatter is more appropriate. This is especially true for abundance matching because the presence of scatter changes the rank order of galaxies. We rank our halos and subhalos by stellar mass, so the rank order will be affected by the assumed amount of scatter. Scatter is typically accounted for by assuming a distribution of stellar mass at fixed halo mass, with the mean value of stellar mass given by a particular SHMR. For example, the B10 scatter that we adopt in this paper is a log-normal distribution with a dispersion $\sigma=0.15$. We implement this scatter by randomly drawing from this distribution when we assign stellar masses to halos and subhalos at the time of accretion. The results shown in Figures 4 and 5 include this scatter.

We repeat our analysis with different dispersions in order to test the sensitivity of our results to uncertainty of the scatter in the SHMR. Figure 6 Shows the effect of changing the SHMR dispersion for one of the models that matches the clustering of $M_{r}<-21$ galaxies: Model 1 with $\epsilon=0.3$. This sample is the most sensitive to scatter because the slope of the SHMR is shallowest for luminous galaxies and thus a given amount of scatter in stellar mass at fixed halo mass translates into a large scatter in halo mass at a fixed stellar mass threshold (see Fig. 3). The top panel of Figure 6] shows the correlation function for three SHMR dispersions: $\sigma=0.1,0.15$, and 0.2 . The bottom panel depicts percent deviations from the fiducial scatter of $\sigma=0.15$. This range is conservative, as it is larger than the errors in scatter quoted in B10 (especially at the low end). The figure demonstrates that changing the scatter has a small effect on $w_{\mathrm{p}}\left(r_{\mathrm{p}}\right)$, in the sense that more scatter generally leads to a lower amplitude of clustering on small scales. Over the range of data points from the Zehavi et al. (2011) data that we model, there is a maximum $\sim 8 \%$ and $\sim 5 \%$ shift in $w_{\mathrm{p}}\left(r_{\mathrm{p}}\right)$ for the $\sigma=0.1$ and $\sigma=0.2$ cases, respectively. This is not a large effect and does not result in a significant change to the best-fit values of $\epsilon$ or $\psi$. Uncertainties in the scatter of the SHMR do not significantly alter our results or our primary conclusions.

\section{LUMINOSITY DEPENDENCE OF SATELLITE GALAXY STELLAR MASS LOSS}

Figures $4 \& 5$ indicate that the best-fit values of both $\epsilon$ and $\psi$ vary with luminosity. We highlight these trends in Figure 7. The left-hand panel summarizes results from Model 1, and demonstrates that the best-fit value of $\epsilon$ drops from $\epsilon=0.8$ for $M_{r}<-18$ galaxies to $\epsilon=0.3$ for $M_{r}<-21$ galaxies. There is a strong luminosity dependence associated with satellite galaxy stellar mass 


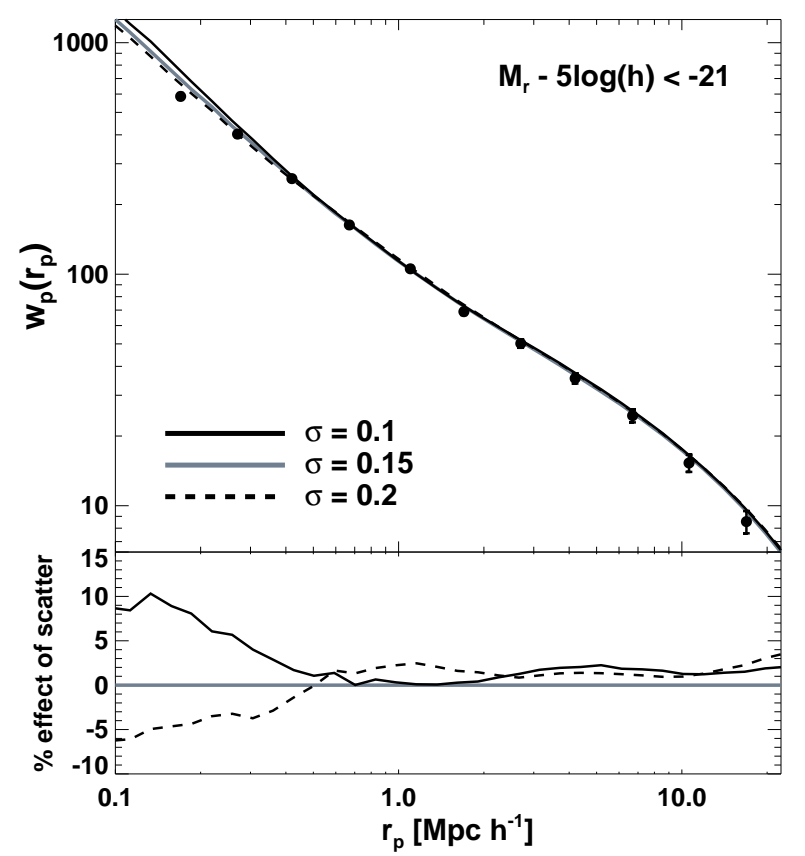

Figure 6. The effect of the scatter in the stellar mass - halo mass relation, on the clustering of a stellar mass threshold galaxy sample. Top panel: The grey curve shows the predicted correlation function from Model 1 with $\epsilon=0.3$ and our assumed scatter of $\sigma=0.15$ dex, given by Behroozi et al. (2010). Solid and dashed black curves show results for changing the scatter to $\sigma=0.1$ dex and 0.2 dex, respectively. Each curve is averaged over three realizations of that amount of scatter. Bottom panel: The percent deviations in the correlation function from the fiducial scatter of $\sigma=0.15$. At the scale of the innermost data point of Zehavi et al. (2011) that we compare our model results to $\left(\sim 0.17 h^{-1} \mathrm{Mpc}\right)$, lowering the scatter to $\sigma=0.1$ increases $w_{\mathrm{p}}\left(r_{\mathrm{p}}\right)$ by $\sim 8 \%$ and increasing the scatter to $\sigma=0.2$ lowers $w_{\mathrm{p}}\left(r_{\mathrm{p}}\right)$ by $\sim 5 \%$. On larger scales the effect is much smaller.

loss, such that relatively low-luminosity satellite galaxies lose more stellar material per unit of dark matter than more luminous satellite galaxies. The right-hand panel of Figure 7 summarizes results from Model 2, which show a consistent trend. The best-fit value of $\psi$ drops from $\psi=$ 0.95 for $M_{r}<-18$ galaxies to $\psi=0.35$ for $M_{r}<-21$ galaxies. Lower values of $\psi$ indicate less stellar mass loss for a given amount of dark matter mass loss. The two models we explore absorb a significant number of subtle effects into simple assumptions. Model 1 and Model 2 differ substantially in detail, yet both analyses indicate that the luminosity dependence of galaxy clustering can be explained if low-luminosity satellite galaxies lose stars more efficiently than luminous galaxies.

What does this result mean? Presumably it means that the stars within luminous galaxies are more tightly bound - relative to their surrounding dark matter - than the stars in less luminous galaxies. This could be due to luminous galaxies having more compact stellar density profiles. In fact, it may be that this result can be explained by the well-known correlation between galaxy luminosity and morphology, whereby luminous galaxies are more likely to be ellipticals than low-luminosity galaxies. For example, Blanton et al. (2003) showed that the mean Sércic index of galaxies roughly doubles from $M_{r}=-18$ to $M_{r}=-21$ (looking at their Fig. 9 and converting $i$ - band to $r$-band magnitudes). We must, of course, keep in mind that these morphology-luminosity correlations apply to all galaxies, of which satellites are only a small portion. It may be that these correlations would vanish in samples containing only satellite galaxies.

An ancillary consideration is the relative size of galaxies compared to the typical (sub)halos they occupy. For instance, using a sample of 140,000 SDSS galaxies, Shen et al. (2003) showed the size distribution (half-light radii) of early- and late-type galaxies as a function of $r$-band luminosity and stellar mass. Examining their Figs. $2 \& 3$, the half-light radius $\bar{R}$ increases roughly by a factor of $\sim 3$ from $M_{r}<-19.25$ to -22.25 for late-type galaxies, and by a factor of $\sim 5$ for early types. However, if we consider the typical halo masses that host these galaxies (see $M_{\min }$ values for $M_{r}<-19$ and -22 from Table 3 of Zehavi et al. 2011), they correspond to virial radii of $\sim 125 h^{-1} \mathrm{kpc}$ and $\sim 750 h^{-1} \mathrm{kpc}$, respectively, a factor of 6 . In other words, at a very approximate level, larger (more luminous) galaxies are slightly deeper within their host halo potential wells than smaller (less luminous) galaxies, and are relatively less susceptible to stellar stripping.

The luminosity trend that we have found demonstrates the power of our modeling approach. We use one observational measurement, galaxy clustering, to shed light on an important physical process that is essentially unobservable: stellar mass loss from satellite galaxies relative to dark matter mass loss. In the next section we show how our models can be used to predict a different observable, the IHL. The IHL is a remnant of stellar mass loss, so it may be used to probe the liberated stellar debris, and thus provide an important cross-check on our results.

\section{PREDICTIONS FOR INTRAHALO LIGHT AT VARYING SCALES}

If stars are freed from their host galaxies they will presumably become part of the IHL. Some of the IHL is a diffuse background, while some may be composed of coherent streams, a prominent example of which is the Sagittarius Stream in our own galaxy (e.g., Ibata et al. 2001; Dohm-Palmer et al. 2001; (Newberg et al. 2002), if the stars were stripped from a subhalo in the past few dynamical times. Observations of the IHL are notoriously difficult, and Gonzalez et al. (2007) argue that the proper quantity to measure is the sum of the light from the host central galaxy (the "brightest halo galaxy" or BHG) and the diffuse intrahalo light component, because it is non-trivial to disentangle the two. Our models assign stellar masses to all halos (both hosts and subhalos), so we can compute the contribution of the BHG to the total stellar mass of the system. This combination is also less subject to theoretical uncertainty because collisions between the BHG and an infalling satellite can disperse stellar mass into the IHL. While parsing of the stellar mass of the BHG and IHL individually is sensitive to the fraction of stellar mass ejected in such collisions, the sum is robust to uncertainties in this process.

We start by investigating the predictions for the $\epsilon=0$ and $\epsilon=1$ cases from Model 1. These extremes were shown in $\S[$ to over- and under-predict the observed clustering, respectively, for each luminosity threshold. In Figure 8, we show the fraction of the total stellar mass 

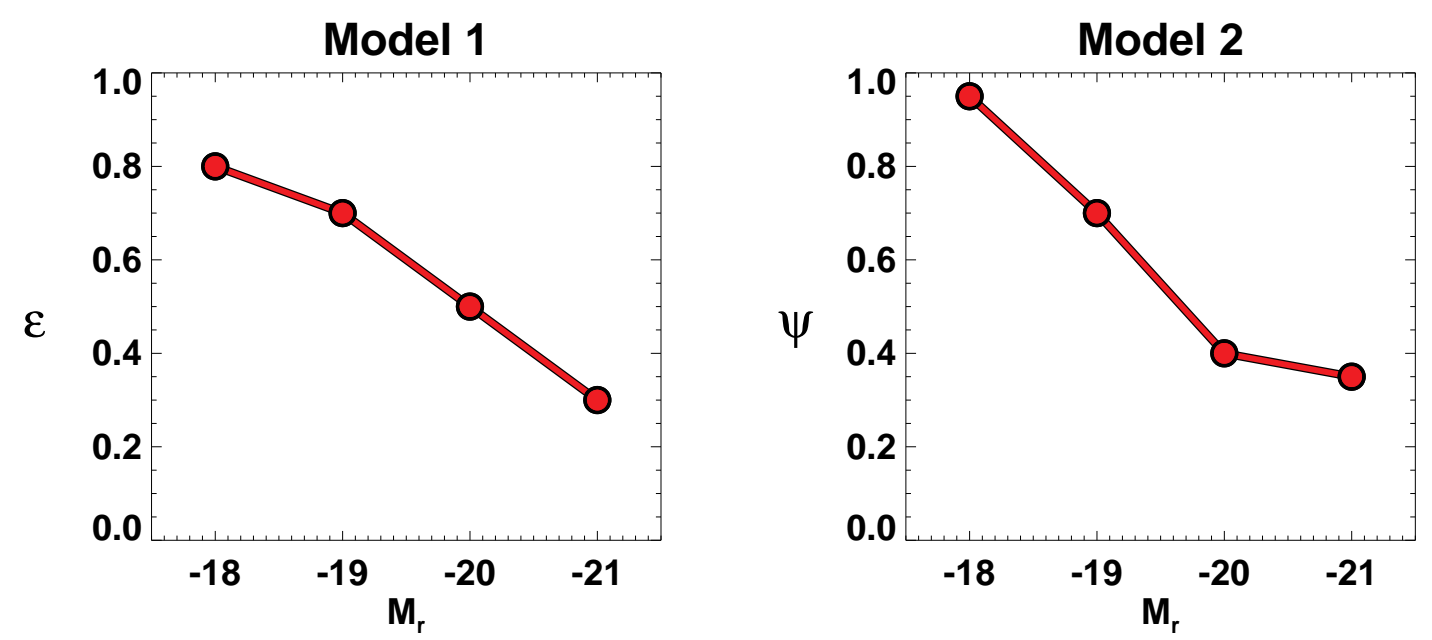

Figure 7. The luminosity dependence of satellite galaxy stellar mass loss. Left panel: The best-fit Model 1 parameter $\epsilon$ as a function of absolute $r$-band magnitude. The decreasing trend of $\epsilon$ with increasing luminosity means that low-luminosity satellite galaxies experience greater stellar mass loss relative to subhalo dark matter mass loss than luminous galaxies. Right panel: The best-fit Model 2 parameter $\psi$ as a function of absolute $r$-band magnitude. The decreasing trend of $\psi$ with increasing luminosity means that low-luminosity satellite galaxies have a greater radius of influence (causing more stellar mass loss) than luminous galaxies. In other words, the same qualitative luminosity trend is seen in both models.
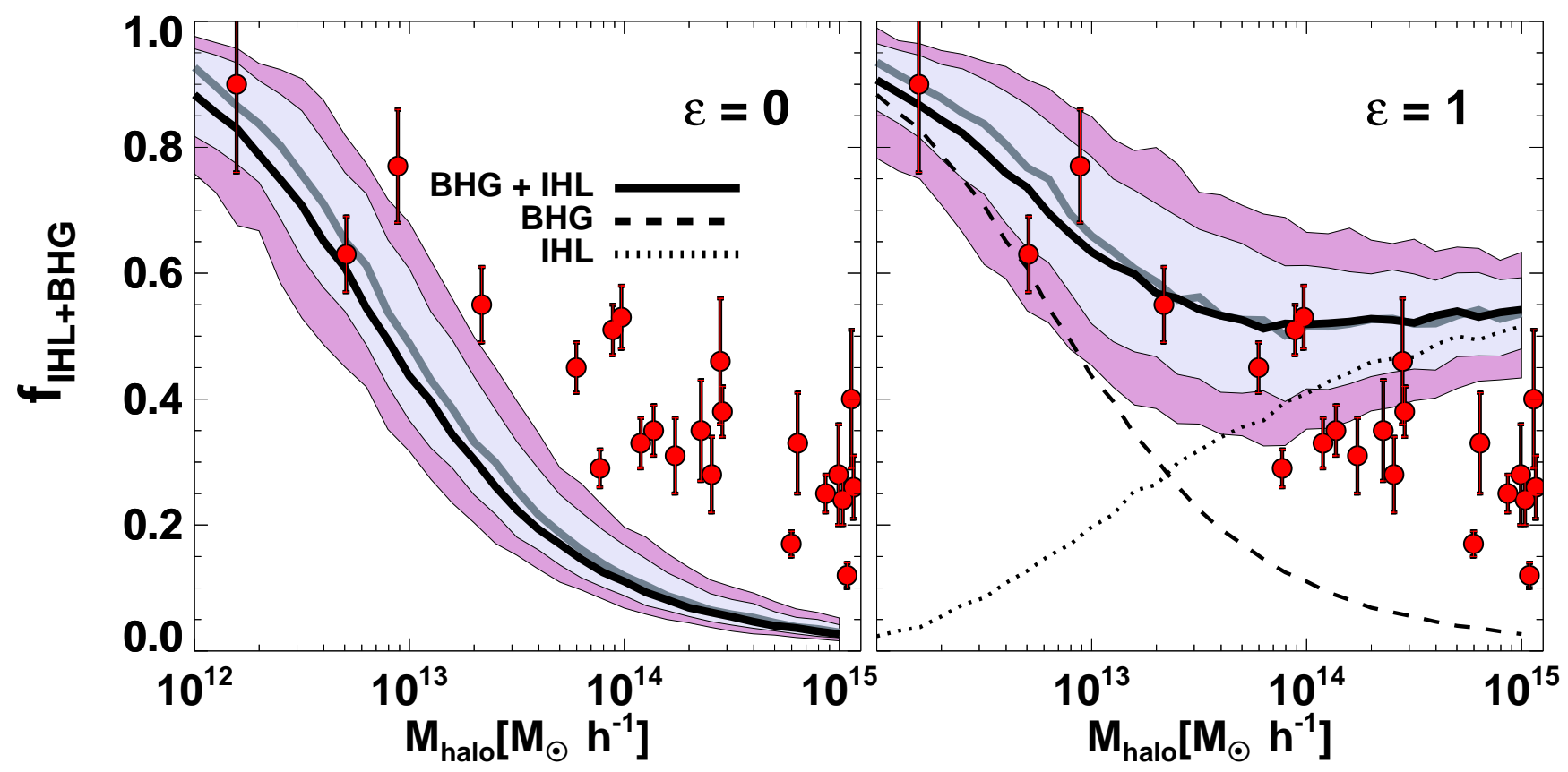

Figure 8. $f_{\mathrm{IHL}+\mathrm{BHG}}$ predictions for the two extreme cases of Model 1: $\epsilon=0$ and 1. $f_{\mathrm{IHL}+\mathrm{BHG}}$ is the combined amount of stellar mass from the brightest halo galaxy (BHG) and the intrahalo light (IHL) divided by the total stellar mass of a system (the BHG, IHL, and the stellar mass still associated with surviving subhalos). Thick solid black curves show the mean $f_{\mathrm{IHL}+\mathrm{BHG}}$ as a function of host halo mass and are computed from 500 model realizations of each host mass, while grey curves represent the median. Light and dark purple shaded regions represent the $68 \%$ and $95 \%$ range of the model distributions. BHG and IHL contributions are shown individually as dashed and dotted curves, respectively. Red points show the Gonzalez et al. (2007) data measurements for comparison. Left panel: $\epsilon=0$ means that no stellar mass loss occurs, resulting in no IHL. As a result, $f_{\mathrm{IHL}+\mathrm{BHG}}$ is strongly under-predicted at cluster scales $\left(M_{\mathrm{halo}} \sim 14-15 h^{-1} M_{\odot}\right)$ relative to the data. Right panel: $\epsilon=1$ means that stellar mass loss occurs at the same rate as subhalo dark matter mass loss. This results in an over-prediction of the IHL. Note: data points are shifted by $\sim 40 \%$ in order to convert the $M_{500}\left(\Delta_{\text {crit }}=500\right)$ masses given in Gonzalez et al. (2007) to our virial masses (using the definition $\Delta_{\text {vir }}=377$ ). 


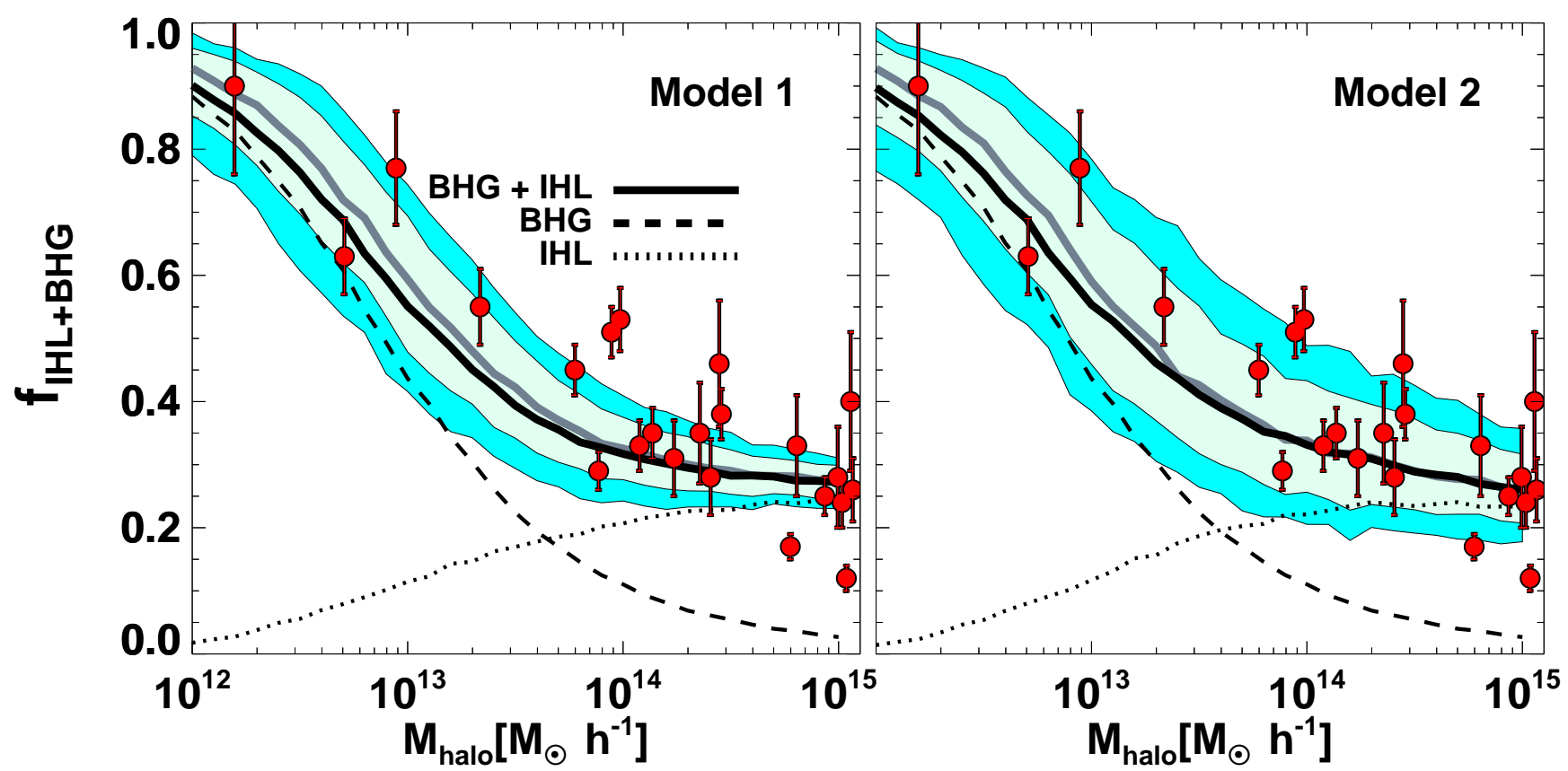

Figure 9. $f_{\mathrm{IHL}+\mathrm{BHG}}$ predictions for our best-fit Model 1 (left panel) and Model 2 (right panel). We compute each subhalo's contribution to the IHL according to its initial stellar mass by using the appropriate best-fit values of $\epsilon$ or $\psi$. For example, a subhalo with a stellar mass that corresponds to a galaxy of absolute $r$-band magnitude equal to -18.5 loses stellar mass according to $\epsilon=0.8$ (in Model 1 ), whereas a subhalo with a stellar mass corresponding to $M_{r}=-20.5$ loses stellar mass according to $\epsilon=0.5$. Shaded regions, curves, and points are as in Fig. 8 Our stellar mass loss models predict that the mean IHL fraction in halos increases from a few percent at the scale of individual galaxies $\left(M_{\text {halo }} \sim 10^{12} h^{-1} M_{\odot}\right)$ to $\sim 20-25 \%$ at cluster scales $\left(M_{\text {halo }} \sim 10^{14}-10^{15} h^{-1} M_{\odot}\right)$. Both models succeed in matching the mean trend observed by Gonzalez et al. (2007). However, Model 1 seems to under-predict the scatter in $f_{\mathrm{IHL}+\mathrm{BHG}}$, whereas Model 2 predicts a scatter that is more consistent with the data measurements. We thus find that this more physically-motivated model for satellite galaxy stellar mass loss is favored over Model 1. 


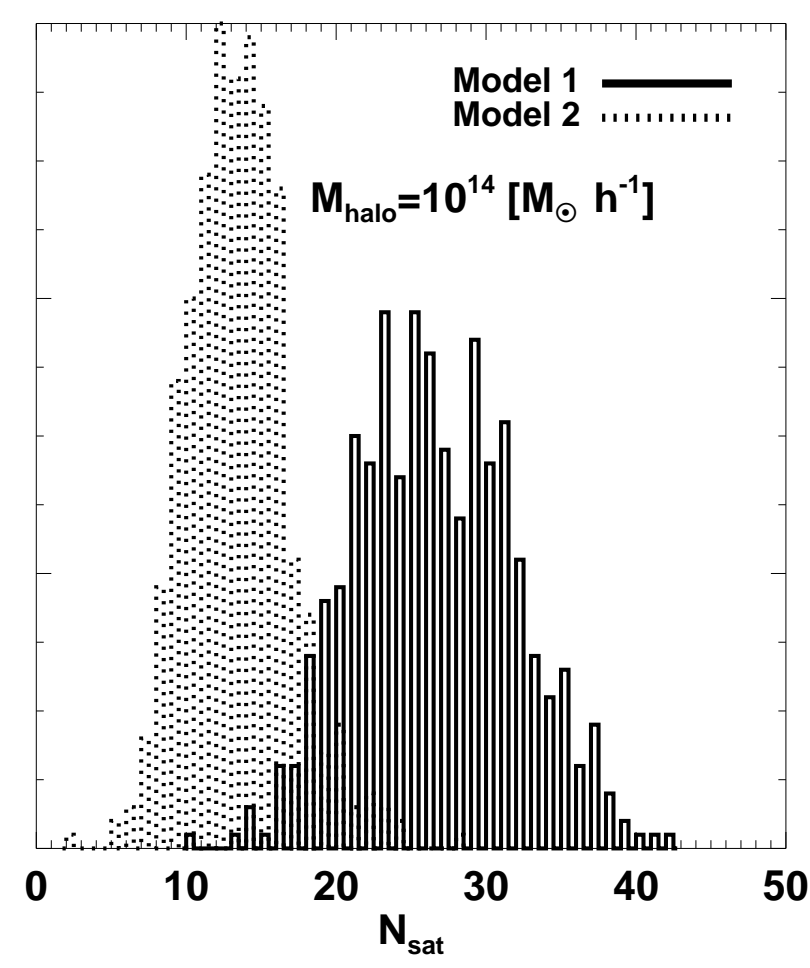

Figure 10. Histograms of the number of satellites that contribute $75 \%$ of the total IHL to a host halo of mass $10^{14} h^{-1} M_{\odot}$ for 500 realizations of Model 1 (solid histogram) and Model 2 (dotted histogram). It is clear that in Model 2 the same amount of IHL comes from a much smaller number of satellites that have each been stripped of more stars than in Model 1. Since fewer satellites contribute to the IHL in Model 2, the Poisson scatter in this model is larger and is evident in the $f_{\mathrm{IHL}+\mathrm{BHG}}$ scatter in Fig. 9

of a system (including stellar mass in satellite galaxies), that is contained within both the IHL and the BHG, $f_{\mathrm{IHL}+\mathrm{BHG}}$, as a function of host halo mass, $M_{\text {halo }}$. The thick solid black curves in Figure 8 show the mean value of $f_{\mathrm{IHL}+\mathrm{BHG}}$ obtained from our 500 realizations of host halos, and grey curves represent the median. The inner and outer shaded regions represent the $68 \%$ and $95 \%$ ranges of the model distributions. The mean individual BHG and IHL contributions are shown distinctly as dashed and dotted curves, respectively. The $f_{\mathrm{IHL}+\mathrm{BHG}}$ data from Gonzalez et al. (2007) are shown as red circles with errorbars, and are the same in both panels.

For $\epsilon=0$ there is no stellar mass loss, and thus no contribution to the total stellar mass of the system from the IHL. The stellar mass of the BHG alone provides a decent description of the data for halo masses below group mass scales, $M_{\text {halo }} \lesssim 10^{13} h^{-1} M_{\odot}$. While less massive systems have been observed to have some detectable IHL (see $\S 1$ ), it is mainly large group- and cluster-size objects that are known to have significant IHL components. It is therefore not surprising that the $\epsilon=0$ model underestimates $f_{\text {IHL+BHG }}$ on these scales.

At the other extreme, the $\epsilon=1$ model assumes that stellar mass is lost with the same efficiency as dark matter. We argued in $\S 4$ that this over-estimates stellar mass loss, so it is not surprising that choosing $\epsilon=1$ leads to an overestimate of $f_{\mathrm{IHL}+\mathrm{BHG}}$ for systems greater than large groups $\left(M_{\text {halo }} \gtrsim 10^{13.5} h^{-1} M_{\odot}\right)$. At lower masses, typical infalling satellites have halo masses below $M_{\mathrm{acc}}^{\mathrm{dm}} \lesssim 10^{11} h^{-1} M_{\odot}$ and bring with them only a small amount of stellar mass (B10). This is the regime of the SHMR where stellar mass rapidly decreases with decreasing halo mass (see Fig. 31). Data are not yet precise enough to be sensitive to the stellar mass carried into the systems by such small, infalling subhalos. Consequently, $f_{\mathrm{IHL}+\mathrm{BHG}} \approx f_{\mathrm{BHG}}$ and can be adequately described in low-mass systems at both the $\epsilon=1$ and $\epsilon=0$ extremes.

We note that in our model predictions for the IHL, we only consider the stellar mass stripped from satellite galaxies that have stellar masses equivalent to $M_{r}<-18$ galaxies and brighter. We ignore stellar mass loss from less luminous (less massive) satellites in the $f_{\mathrm{IHL}+\mathrm{BHG}}$ calculation. However, the stellar mass locked up in these lower mass "uncounted" subhalos only amounts to $\sim 3 \%$ of the total stellar mass within a given host halo, so neglecting them has a negligible effect on our $f_{\mathrm{IHL}+\mathrm{BHG}}$ model predictions. We also note that in Figure 8 we have shifted the data points to higher masses by $\sim 40 \%$ in order to convert the $M_{500}\left(\Delta_{\text {crit }}=500\right)$ masses given in Gonzalez et al. (2007) to our virial masses (using the definition $\Delta_{\text {vir }}=377$ ). We assumed an NFW profile to perform this conversion.

Having shown that these two extreme cases are not able to describe the data accurately, we turn to the predictions of our models. To make $f_{\mathrm{IHL}+\mathrm{BHG}}$ predictions at a given host halo mass scale we need to assign each (sub)halo an $\epsilon$ value for Model 1 and a $\psi$ value for Model 2. Let us examine just the Model 1 case for simplicity. When we found the best-fit value of $\epsilon$ for each luminosity threshold sample in $\S 5$, we also determined a final stellar mass threshold for the sample through abundance matching. For example, the $M_{r}<-20$ sample was found to correspond to halos and subhalos with final stellar masses greater than some value $M_{\text {fin, } 20}^{*}$, where final stellar masses are predicted using $\epsilon=0.5$. The $M_{r}<-21$ sample corresponds to stellar masses greater than $M_{\text {fin, } 21}^{*}$, where final stellar masses are predicted using $\epsilon=0.3$, and so on. We assign each subhalo a value of $\epsilon$ using these stellar mass thresholds. For example, if a subhalo has a stellar mass at accretion that falls between $M_{\text {fin, } 20}^{*}$ and $M_{\text {fin, } 21}^{*}$, we assign it $\epsilon=0.5$. If, instead, it has a mass between $M_{\text {fin,19 }}^{*}$ and $M_{\text {fin,20 }}^{*}$, we assign it $\epsilon=0.7$. It is awkward to use the stellar mass at accretion to assign subhalos to ranges in $M_{\text {fin }}^{*}$, but we cannot estimate final stellar masses without having a value of $\epsilon$ in the first place, so we are stuck with this approximate method. We follow the same process for Model 2 by choosing the appropriate $\psi$ values. We emphasize that these $\epsilon$ and $\psi$ values are not "tuned" to agree with the $f_{\mathrm{IHL}+\mathrm{BHG}}$ observations, rather are genuine predictions that result from parameter values found from matching to clustering data.

Figure 9 shows the Model 1 (left panel) and Model 2 (right panel) $f_{\mathrm{IHL}+\mathrm{BHG}}$ predictions, as well as the individual IHL and BHG contributions. While $f_{\mathrm{IHL}+\mathrm{BHG}}$ rapidly decreases with increasing host halo mass, the contribution to $f_{\mathrm{IHL}+\mathrm{BHG}}$ from the IHL increases. This supports a picture in which galaxy-mass halos have very few luminous satellite galaxies and little IHL, so the stellar masses of these systems are dominated by the 
BHGs. Galaxy size halos accrete the majority of their mass from small subhalos that have very high mass-tolight ratios (Purcell et al. 2007). Therefore, shredded satellites deposit very little stellar mass into the IHL. The number of large satellites increases with host halo mass. The larger and more common subhalos within larger host halos may form stars more efficiently (have lower mass-to-light ratios) and provide a source for developing significant IHL. Both models find that host masses of $M_{\text {host }} / h^{-1} M_{\odot}=10^{12}, 10^{13}, 10^{14}$, and $10^{15}$, contain mean IHL fractions of $f_{\mathrm{IHL}} \sim 2 \%, 10 \%, 20 \%$, and $25 \%$, respectively. This is in good agreement with several previous studies, as we discuss in $\S 8$. The IHL thus provides an independent check of our stellar mass loss results.

Figure 9] contains a comparison between our model predictions and the Gonzalez et al. (2007) data. Both models predict mean $f_{\mathrm{IHL}+\mathrm{BHG}}$ fractions that are consistent with the observations 6 Interestingly, we find that the scatter predicted by Model 1 is substantially smaller than it is for Model 2 and it appears to be inconsistent with the scatter found by Gonzalez et al. (2007). On the other hand, Model 2 is more successful in reproducing the observed scatter in $f_{\mathrm{IHL}+\mathrm{BHG}}$. To quantify this, we calculate the probability that the scatter in the Gonzalez et al. (2007) data points could result from the model distributions. We do this as follows. First, we restrict the comparison to the high mass regime (the last 8 data points) because we wish to investigate the scatter at fixed mass (if we include lower mass points, the measured scatter will be affected by the mean trend of $f_{\text {IHL }+ \text { BHG }}$ vs. mass). We next create a model data set by choosing a single model realization of a host halo (out of the 500) for each Gonzalez et al. (2007) data point, at the appropriate halo masses. This leads to 8 model values of $f_{\mathrm{IHL}+\mathrm{BHG}}$. We then add a Gaussian random error to these values, using the corresponding Gonzalez et al. (2007) errorbars. To fully sample the distribution of data sets that could arise from our models, we repeat this process 10,000 times. Finally, we measure the weighted standard deviation of $f_{\mathrm{IHL}}+\mathrm{BHG}$ values for the observed data, as well as each of the 10,000 model data sets. We find that in Model 1 , only $0.8 \%$ of the model data sets have a scatter that exceeds that of the Gonzalez et al. (2007) data, whereas in Model 2, 25\% of the model data sets have a larger scatter.

This statistical test indicates that Model 1 should be rejected (at the $2.7 \sigma$ level) in favor of Model 2, an indication that is not entirely surprising given that Model 2 accounts for delayed stellar mass loss that is to be expected on physical grounds. Model 1 assumes that stellar mass loss is simply proportional to subhalo mass loss, so the scatter predicted by this model must be primarily caused by the scatter in the total amount of dark matter mass loss, with a secondary amount of scatter coming from the SHMR. However, the "all or nothing" action provided by the radius of influence in Model 2 somehow amplifies this scatter. Upon closer investigation, we find that the difference in scatter between the two models arises from simple Poisson statistics. Figure 10 shows the

6 We note that this discussion assumes that the Gonzalez et al. (2007) data are accurate, whereas they may be systematically overestimated due to an assumed constant mass-to-light ratio (Leauthaud et al. 2011). distribution of the number of satellites that contribute $75 \%$ of the IHL to a host halo of mass $10^{14} h^{-1} M_{\odot}$, measured from 500 realizations for both Model 1 (solid histogram) and Model 2 (dotted histogram). It is clear that in Model 2 the same amount of IHL comes from a much smaller number of satellites that have each been stripped of more stars than in Model 1 . This is a direct result of the nature of Model 2: most subhalos do not lose stars, but if they do, it is at a high rate. Since fewer satellites contribute to the IHL in Model 2, the Poisson scatter in this model is larger and is manifested in the greater $f_{\mathrm{IHL}+\mathrm{BHG}}$ scatter seen in Fig. 9.

\section{SUMMARY \& DISCUSSION}

While galaxy formation is complex, we have shown in this paper that an understanding of the fates of satellite galaxies provides key insight into the galaxy formation puzzle. Satellite galaxies live extremely tumultuous lives. Their spatial clustering can shed light on how they lose stellar mass and contribute to building the IHL over a large range of host halo mass scales. Part of our motivation for using galaxy clustering to constrain stellar mass loss stems from possible shortcomings in the commonly-employed abundance matching technique (e.g., Wetzel \& White 2010; Yang et al. 2011a, Reddick et al., in prep.), which suggest natural generalizations with the gross form of the particular models we study. The standard method of abundance matching involves mapping galaxies to halos by assuming a relationship between halo (and subhalo) mass (or circular velocity) and a galaxy property (luminosity or stellar mass) at a particular time. A galaxy luminosity assignment according to halo mass at the time the halo was first accreted onto a larger system provides a very useful description of known galaxy clustering properties (e.g., Conroy et al. 2006). However, this type of assignment implies that no stellar mass loss will occur. This is contrary to many aspects of galaxy, group, and cluster evolution, including the prevailing paradigm in which the IHL is produced by stellar mass liberated from infalling galaxies by interactions in the group environment (e.g., Conroy et al. 2007; Purcell et al. 2007; Yang et al. 2009). Abundance matching is also sensitive to the resolution of the cosmological simulation on which it is implemented. Subhalos are artificially destroyed by falling below the numerical resolution limit and immediately removed from the galaxy population irrespective of their circular velocities at their accretion times. This can lead to differing predictions for the small-scale clustering of galaxies of a particular luminosity among simulations with a fixed mapping of halo size onto luminosity.

These considerations persuaded us to explore simple models that associate stellar masses to subhalo masses and constrain satellite galaxy stellar mass loss by matching to clustering data. We implemented two distinct models. Model 1 related the fractional amount of stellar mass loss to that of the subhalo dark matter mass loss from the time of accretion until the final redshift through a single free parameter $\epsilon$. Model 2 was introduced to mimic a "lag" in stellar mass loss wherein the satellite galaxy at the core of the subhalo experiences no stellar mass loss until the subhalo has been stripped of sufficient dark matter on its periphery. Stellar mass loss in this model only occurs once dark matter has been lost 
interior to a radius of influence of the satellite galaxy. We introduced the free parameter $\psi$ that dictated how the radius of influence could expand or shrink allowing for more or less stellar mass loss. We calculated the values of $\epsilon$ and $\psi$ that matched the observed clustering of SDSS galaxies over a large range of luminosity threshold samples. Matching to the observed clustering at each luminosity threshold directly informs us of the amount of stellar mass lost from satellite galaxies and, thus, will comprise the IHL.

This procedure enables predictions for the amount of IHL or IHL+BHG compared to the total stellar mass of a host system of a given mass ( $f_{\mathrm{IHL}}$ and $f_{\text {IHL }+ \text { BHG }}$, respectively). Observationally, it has been established that the amount of diffuse material is nearly negligible at the scale of individual galaxies (e.g., Sackett et al. 1994; Morrison et al. 1997; Weil et al. 1997; Lequeux et al. 1998; Abe et al. 1999; Zibetti \& Ferguson 2004; Bailin et al. 2011). For example, M33, which is $\sim 10^{11} M_{\odot}$, has been estimated to have an $f_{\mathrm{IHL}} \leq 1 \%$ (McConnachie et al. 2006; Hood et al. 2007). Our own Milky Way is thought to have an $f_{\mathrm{IHL}} \sim 1 \%$ (e.g., Morrison 1993; Wetterer \& McGraw 1996; Chiba \& Beers 2000; Ivezić et al. 2000; Morrison et al. 2000; Yannv et al. 2000; Siegel et al. 2002) or $\sim 2 \%$ when including the stars from the Sagittarius stream (Law et al. 2005). Our analog M31 has an $f_{\text {IHL }} \sim 2-5 \%$ (Irwin et al. 2005; Guhathakurta et al. 2005; Chapman et al. 2006; Kalirai et al. 2006). Recently, Bailin et al. (2011) showed that NGC 235, which is of comparable luminosity to the Milky Way and M31, has $f_{\mathrm{IHL}} \sim 6 \%$. On group scales, $\sim 10^{13}-10^{14} M_{\odot}$, measured $f_{\mathrm{IHL}}$ percentages tend to be higher than on galaxy scales, though there can be considerable variation from group to group (e.g., $f_{\mathrm{IHL}}$ on the order of a few percent: Feldmeier et al. 2001; Castro-Rodríguez et al. 2003; Feldmeier 2006, $f_{\mathrm{IHL}} \sim 5-30 \%$ : Da Rocha \& Mendes de Oliveira 2005; Aguerri et al. 2006; Da Rocha et al. 2008, and as high as $f_{\mathrm{IHL}} \sim 45 \%$ : White et al. 2003; McGee \& Balogh 2010). For clusters scales, IHL fractions are typically much higher. There is still substantial scatter, but $f_{\mathrm{IHL}}$ values range from $\sim 10-40 \%$ (Melnick et al. 1977; Thuan \& Kormendv 1977; Uson et al. 1991; Bernstein et al. 1995; Calcáneo-Roldán et al. 2000; Lin \& Mohr 2004; Mihos et al. |2005; Zibetti et al. 2005; Krick et al. 2006; Seigar et al. 2007). As discussed in 87. Gonzalez et al. (2007) posit that the relevant quantity is $f_{\mathrm{IHL}+\mathrm{BHG}}$, because it can be difficult to disentangle the stellar mass associated with central galaxy from the diffuse IHL component. It has been found that $f_{\mathrm{IHL}+\mathrm{BHG}} \sim 30 \%$ on average on cluster scales (Zibetti et al. 2005; Gonzalez et al. 2007; Pierini et al. 2008; Toledo et al. 2011).

Our modeling of satellite galaxy stellar mass loss has yielded the following principal results and conclusions.

- Abundance matching with $\epsilon=0$ and $\psi=0$ ("traditional" abundance matching, akin to using the subhalo mass at the time of infall) over-predicts the correlation function on small scales $(\lesssim 1 \mathrm{Mpc})$ for each luminosity threshold sample.

- For each luminosity threshold, we found both the $\epsilon$ and $\psi$ value that predicted a correlation function that matched the data. We found that $\epsilon$ and $\psi$ were decreasing functions of luminosity. This means that low-luminosity satellite galaxies are more efficiently stripped of stellar material than luminous satellites.

- Our predictions for $f_{\mathrm{IHL}}$ from both Model 1 and Model 2 are in good agreement with observational studies over an enormous range of host halo mass scales. For host masses of $10^{12}$ (individual galaxy scale), $10^{13}-10^{14}$ (group to small cluster scales), and $10^{14}-10^{15}$ (cluster scales), we find mean IHL fractions of a few percent, $10-20 \%$, and $20-25 \%$, respectively. These are in accord with previous observational studies, though there is substantial scatter in the $f_{\mathrm{IHL}}$ measurements in the literature.

- The more physically-motivated Model 2 is consistent with the $f_{\mathrm{IHL}+\mathrm{BHG}}$ measurements of Gonzalez et al. (2007) from small group scales all the way through cluster mass systems. The scatter in the Model 2 results is comparable to the scatter observed in the data. On the contrary, the scatter among $f_{\mathrm{IHL}+\mathrm{BHG}}$ values predicted by Model 1 is insufficient to describe the scatter among the observed systems. This suggests that Model 1, in which stellar mass loss occurs in proportion to dark matter mass loss, can be rejected by current data.

We have shown that galaxy clustering can be used as a powerful tool to understand how satellite galaxies lose stellar mass. We have found the interesting result that low-luminosity galaxies lose more stellar mass relative to subhalo dark matter mass loss than luminous galaxies. Moreover, we were able to predict current IHL observations and thus further constrain our stellar mass loss models. These results show that our modeling framework can mitigate potential problems associated with the conventional abundance matching approach. Also, our approach is generalizable. It allows for the flexibility to make more detailed predictions within this framework (for instance, examining the photometric properties of the IHL as compared to galaxies).

In Paper II, we will take advantage of clustering measurements at high redshifts, allowing us to study the evolution of satellite galaxy stellar mass loss as a function of time. This will enable us to make predictions for the build-up of the IHL over cosmic time. A possible extension of this program is to also explore stellar massselected threshold samples directly rather than the luminosity threshold samples we have used in this paper. This is a natural choice for refining this class of studies because dynamical models most directly treat stellar mass loss.

A further useful avenue to pursue as a result of this work will be to connect detailed theoretical models of stellar mass loss in individual galaxies more directly to the statistical models of stellar mass loss that can be explored with large-scale survey data, such as we have done. Models of stellar mass loss that are consistent with survey data must also be representative of detailed dynamical models of stellar mass loss. It will be very interesting to develop a set of simple, yet powerful models 
for the build-up and dispersal of the stellar mass in satellite galaxies that simultaneously describe the evolution of galaxy clustering and intrahalo light over cosmic time.

\section{ACKNOWLEDGMENTS}

We thank Chris Purcell and Jeff Newman for insightful discussions. The work of DFW and AAB is supported by Vanderbilt University, the Alfred P. Sloan Foundation, and the National Science Foundation (NSF) through grant AST-1109789. The work of ARZ is funded by the Pittsburgh Particle physics, Astrophysics, and Cosmology Center (PITT PACC) at the University of Pittsburgh and by the NSF through grants AST-0806367 and AST1108802 .

\section{REFERENCES}

Abe, F., et al. 1999, AJ, 118, 261

Aguerri, J. A. L., Castro-Rodríguez, N., Napolitano, N., Arnaboldi, M., \& Gerhard, O. 2006, A\&A, 457, 771

Bailin, J., Bell, E. F., Chappell, S. N., Radburn-Smith, D. J., \& de Jong, R. S. 2011, ApJ, 736, 24

Behroozi, P. S., Conroy, C., \& Wechsler, R. H. 2010, ApJ, 717, 379

Behroozi, P. S., Wechsler, R. H., \& Wu, H.-Y. 2011, ArXiv e-prints

Bell, E. F., \& de Jong, R. S. 2001, ApJ, 550, 212

Benson, A. J. 2005, MNRAS, 358, 551

Berlind, A. A., \& Weinberg, D. H. 2002, ApJ, 575, 587

Bernstein, G. M., Nichol, R. C., Tyson, J. A., Ulmer, M. P., \& Wittman, D. 1995, AJ, 110, 1507

Berrier, J. C., Bullock, J. S., Barton, E. J., Guenther, H. D., Zentner, A. R., \& Wechsler, R. H. 2006, ApJ, 652, 56

Blanton, M. R., et al. 2003, ApJ, 594, 186

Blumenthal, G. R., Faber, S. M., Flores, R., \& Primack, J. R. 1986, ApJ, 301, 27

Bond, J. R., Cole, S., Efstathiou, G., \& Kaiser, N. 1991, ApJ, 379,440

Bryan, G. L., \& Norman, M. L. 1998, ApJ, 495, 80

Byrd, G., \& Valtonen, M. 1990, ApJ, 350, 89

Calcáneo-Roldán, C., Moore, B., Bland-Hawthorn, J., Malin, D., \& Sadler, E. M. 2000, MNRAS, 314, 324

Castro-Rodríguez, N., Aguerri, J. A. L., Arnaboldi, M., Gerhard, O., Freeman, K. C., Napolitano, N. R., \& Capaccioli, M. 2003, A\&A, 405, 803

Chandrasekhar, S. 1943, ApJ, 97, 255

Chapman, S. C., Ibata, R., Lewis, G. F., Ferguson, A. M. N., Irwin, M., McConnachie, A., \& Tanvir, N. 2006, ApJ, 653, 255

Chiba, M., \& Beers, T. C. 2000, AJ, 119, 2843

Conroy, C., \& Wechsler, R. H. 2009, ApJ, 696, 620

Conroy, C., Wechsler, R. H., \& Kravtsov, A. V. 2006, ApJ, 647, 201

-2007, ApJ, 668, 826

Cooray, A., \& Sheth, R. 2002, Phys. Rep., 372,

Da Rocha, C., \& Mendes de Oliveira, C. 2005, MNRAS, 364, 1069

Da Rocha, C., Ziegler, B. L., \& Mendes de Oliveira, C. 2008, MNRAS, 388, 1433

Davis, M., \& Peebles, P. J. E. 1983, ApJ, 267, 465

Del Popolo, A.. \& Kroupa, P. 2009, A\&A, 502, 733

Diemand, J., Kuhlen, M., \& Madau, P. 2007, ApJ, 667, 859

Diemand, J., Moore, B., \& Stadel, J. 2004, MNRAS, 353, 624

Dohm-Palmer, R. C., et al. 2001, ApJ, 555, L37

Faltenbacher, A., \& Mathews, W. G. 2005, MNRAS, 362, 498

Feldmeier, J. J. 2006, in IAU Symposium, Vol. 234, Planetary Nebulae in our Galaxy and Beyond, ed. M. J. Barlow \& R. H. Méndez, 33-40

Feldmeier, J. J., Durrell, P. R., Ciardullo, R., \& Jacoby, G. H. 2001, ArXiv Astrophysics e-prints

Fukushige, T., Kawai, A., \& Makino, J. 2004, ApJ, 606, 625

Gallagher, III, J. S., \& Ostriker, J. P. 1972, AJ, 77, 288

Gan, J., Kang, X., van den Bosch, F. C., \& Hou, J. 2010, MNRAS, 408, 2201

Ghigna, S., Moore, B., Governato, F., Lake, G., Quinn, T., \& Stadel, J. 2000, ApJ, 544, 616
Giocoli, C., Pieri, L., Tormen, G., \& Moreno, J. 2009, MNRAS, 395,1620

Giocoli, C., Tormen, G., \& van den Bosch, F. C. 2008, MNRAS, 386,2135

Gnedin, O. Y. 2003, ApJ, 589, 752

Gnedin, O. Y., Kravtsov, A. V., Klypin, A. A., \& Nagai, D. 2004, ApJ, 616, 16

Gonzalez, A. H., Zaritsky, D., \& Zabludoff, A. I. 2007, ApJ, 666, 147

Governato, F., et al. 2012, MNRAS, 2697

Guhathakurta, P., Ostheimer, J. C., Gilbert, K. M., Rich, R. M., Majewski, S. R., Kalirai, J. S., Reitzel, D. B., \& Patterson, R. J. 2005, ArXiv Astrophysics e-prints

Guo, Q., White, S., Li, C., \& Boylan-Kolchin, M. 2010, MNRAS, 404, 1111

Hashimoto, Y., Funato, Y., \& Makino, J. 2003, ApJ, 582, 196

Hayashi, E., Navarro, J. F., Taylor, J. E., Stadel, J., \& Quinn, T. 2003, ApJ, 584, 541

Hood, M., Smecker-Hane, T., Teig, M., Ferguson, A. M. N., \& Irwin, M. J. 2007, in Astronomical Society of the Pacific Conference Series, Vol. 374, From Stars to Galaxies: Building the Pieces to Build Up the Universe, ed. A. Vallenari, R. Tantalo, L. Portinari, \& A. Moretti, 281-+

Ibata, R., Irwin, M., Lewis, G. F., \& Stolte, A. 2001, ApJ, 547, L133

Irwin, M. J., Ferguson, A. M. N., Ibata, R. A., Lewis, G. F., \& Tanvir, N. R. 2005, ApJ, 628, L105

Ivezić, Ž., et al. 2000, AJ, 120, 963

Kalirai, J. S., et al. 2006, ApJ, 648, 389

Kazantzidis, S., Mayer, L., Mastropietro, C., Diemand, J., Stadel, J., \& Moore, B. 2004, ApJ, 608, 663

Kitzbichler, M. G., \& White, S. D. M. 2008, MNRAS, 391, 1489

Klypin, A., Gottlöber, S., Kravtsov, A. V., \& Khokhlov, A. M. 1999, ApJ, 516, 530

Komatsu, E., et al. 2011, ApJS, 192, 18

Koushiappas, S. M., Zentner, A. R., \& Kravtsov, A. V. 2010, ArXiv e-prints

Kravtsov, A. V., Berlind, A. A., Wechsler, R. H., Klypin, A. A., Gottlöber, S., Allgood, B., \& Primack, J. R. 2004a, ApJ, 609, 35

Kravtsov, A. V., Gnedin, O. Y., \& Klypin, A. A. 2004b, ApJ, 609,482

Krick, J. E., Bernstein, R. A., \& Pimbblet, K. A. 2006, AJ, 131, 168

Lacey, C., \& Cole, S. 1993, MNRAS, 262, 627

$$
\text { 1994, MNRAS, 271, } 676
$$

Law, D. R., Johnston, K. V., \& Majewski, S. R. 2005, ApJ, 619, 807

Leauthaud, A., et al. 2011, ArXiv e-prints

Lequeux, J., Combes, F., Dantel-Fort, M., Cuillandre, J., Fort, B., \& Mellier, Y. 1998, A\&A, 334, L9

Lin, Y., \& Mohr, J. J. 2004, ApJ, 617, 879

Mackey, A. D., et al. 2010, ApJ, 717, L11

McConnachie, A. W., Chapman, S. C., Ibata, R. A., Ferguson, A. M. N., Irwin, M. J., Lewis, G. F., Tanvir, N. R., \& Martin, N. 2006, ApJ, 647, L25

McGee, S. L., \& Balogh, M. L. 2010, MNRAS, 403, L79

Melnick, J., Hoessel, J., \& White, S. D. M. 1977, MNRAS, 180, 207

Merritt, D. 1983, ApJ, 264, 24

Mihos, J. C., Harding, P., Feldmeier, J., \& Morrison, H. 2005, ApJ, 631, L41

More, S., van den Bosch, F. C., Cacciato, M., Mo, H. J., Yang, X., \& Li, R. 2009, MNRAS, 392, 801

Morrison, H. L. 1993, AJ, 106, 578

Morrison, H. L., Mateo, M., Olszewski, E. W., Harding, P., Dohm-Palmer, R. C., Freeman, K. C., Norris, J. E., \& Morita, M. 2000, AJ, 119, 2254

Morrison, H. L., Miller, E. D., Harding, P., Stinebring, D. R., \& Boroson, T. A. 1997, AJ, 113, 2061

Moster, B. P., Somerville, R. S., Maulbetsch, C., van den Bosch,

F. C., Macciò, A. V., Naab, T., \& Oser, L. 2010, ApJ, 710, 903

Murante, G., et al. 2004, ApJ, 607, L83

Nagai, D., \& Kravtsov, A. V. 2005, ApJ, 618, 557

Navarro, J. F., Frenk, C. S., \& White, S. D. M. 1997, ApJ, 490, 493 
Neistein, E., Li, C., Khochfar, S., Weinmann, S. M., Shankar, F., \& Boylan-Kolchin, M. 2011, ArXiv e-prints

Newberg, H. J., et al. 2002, ApJ, 569, 245

Oguri, M., \& Lee, J. 2004, MNRAS, 355, 120

Peñarrubia, J., \& Benson, A. J. 2005, MNRAS, 364, 977

Peñarrubia, J., Benson, A. J., Walker, M. G., Gilmore, G., McConnachie, A. W., \& Mayer, L. 2010, MNRAS, 406, 1290

Peñarrubia, J., McConnachie, A. W., \& Navarro, J. F. 2008a, ApJ, 672, 904

Peñarrubia, J., Navarro, J. F., \& McConnachie, A. W. 2008b, ApJ, 673, 226

Peacock, J. A., \& Smith, R. E. 2000, MNRAS, 318, 1144

Pierini, D., Zibetti, S., Braglia, F., Böhringer, H., Finoguenov, A., Lynam, P. D., \& Zhang, Y. 2008, A\&A, 483, 727

Purcell, C. W., Bullock, J. S., \& Zentner, A. R. 2007, ApJ, 666, 20 - 2008, MNRAS, 391, 550

Reed, D., Governato, F., Verde, L., Gardner, J., Quinn, T., Stadel, J., Merritt, D., \& Lake, G. 2005, MNRAS, 357, 82

Romanowsky, A. J., Strader, J., Brodie, J. P., Mihos, J. C., Spitler, L. R., Forbes, D. A., Foster, C., \& Arnold, J. A. 2012, ApJ, 748, 29

Rudick, C. S., Mihos, J. C., Frey, L. H., \& McBride, C. K. 2009, ApJ, 699, 1518

Rudick, C. S., Mihos, J. C., \& McBride, C. K. 2011, ApJ, 732, 48

Ryden, B. S., \& Gunn, J. E. 1987, ApJ, 318, 15

Sackett, P. D., Morrisoni, H. L., Harding, P., \& Boroson, T. A. 1994, Nature, 370, 441

Scoccimarro, R., Sheth, R. K., Hui, L., \& Jain, B. 2001, ApJ, 546, 20

Seigar, M. S., Graham, A. W., \& Jerjen, H. 2007, MNRAS, 378, 1575

Shen, S., Mo, H. J., White, S. D. M., Blanton, M. R., Kauffmann, G., Voges, W., Brinkmann, J., \& Csabai, I. 2003, MNRAS, 343, 978

Siegel, M. H., Majewski, S. R., Reid, I. N., \& Thompson, I. B. 2002, ApJ, 578, 151

Simha, V., Weinberg, D., Dave, R., Fardal, M., Katz, N., \& Oppenheimer, B. D. 2010, ArXiv e-prints

Skibba, R. A., Sheth, R. K., \& Martino, M. C. 2007, MNRAS, 382,1940

Somerville, R. S., \& Kolatt, T. S. 1999, MNRAS, 305, 1

Sommer-Larsen, J. 2006, MNRAS, 369, 958

Tasitsiomi, A., Kravtsov, A. V., Wechsler, R. H., \& Primack, J. R. 2004, ApJ, 614, 533

Taylor, J. E., \& Babul, A. 2004, MNRAS, 348, 811

- 2005a, MNRAS, 364, 515

- 2005b, MNRAS, 364, 535

Thuan, T. X., \& Kormendy, J. 1977, PASP, 89, 466
Toledo, I., Melnick, J., Selman, F., Quintana, H., Giraud, E., \& Zelaya, P. 2011, MNRAS, 414, 602

Uson, J. M., Boughn, S. P., \& Kuhn, J. R. 1991, ApJ, 369, 46

Vale, A., \& Ostriker, J. P. 2004, MNRAS, 353, 189 2006, MNRAS, 371, 1173

van den Bosch, F. C., Tormen, G., \& Giocoli, C. 2005, MNRAS, 359,1029

van den Bosch, F. C., et al. 2007, MNRAS, 376, 841

Wang, L., \& Jing, Y. P. 2010, MNRAS, 402, 1796

Wang, L., Li, C., Kauffmann, G., \& De Lucia, G. 2006, MNRAS, 371,537

Warren, M. S., Abazajian, K., Holz, D. E., \& Teodoro, L. 2006, ApJ, 646, 881

Watson, D. F., Berlind, A. A., \& Zentner, A. R. 2011, ApJ, 738, 22

Wechsler, R. H., Bullock, J. S., Primack, J. R., Kravtsov, A. V., \& Dekel, A. 2002, ApJ, 568, 52

Weil, M. L., Bland-Hawthorn, J., \& Malin, D. F. 1997, ApJ, 490, 664

Wetterer, C. J., \& McGraw, J. T. 1996, AJ, 112, 1046

Wetzel, A. R., Tinker, J. L., \& Conroy, C. 2011, ArXiv e-prints

Wetzel, A. R., \& White, M. 2010, MNRAS, 403, 1072

White, P. M., Bothun, G., Guerrero, M. A., West, M. J., \&

Barkhouse, W. A. 2003, ApJ, 585, 739

White, S. D. M., \& Rees, M. J. 1978, MNRAS, 183, 341

Willman, B., Governato, F., Wadsley, J., \& Quinn, T. 2004, MNRAS, 355, 159

Yang, X., Mo, H. J., \& van den Bosch, F. C. 2003, MNRAS, 339, 1057

-2008, ApJ, 676, 248

- 2009, ApJ, 693, 830

Yang, X., Mo, H. J., van den Bosch, F. C., Zhang, Y., \& Han, J. 2011a, ArXiv e-prints

Yang, X., Mo, H. J., Zhang, Y., \& van den Bosch, F. C. 2011b, ApJ, 741, 13

Yanny, B., et al. 2000, ApJ, 540, 825

York, D. G., et al. 2000, AJ, 120, 1579

Zappacosta, L., Buote, D. A., Gastaldello, F., Humphrey, P. J., Bullock, J., Brighenti, F., \& Mathews, W. 2006, ApJ, 650, 777

Zehavi, I., et al. 2004, ApJ, 608, 16

- 2011, ApJ, 736, 59

Zentner, A. R. 2007, International Journal of Modern Physics D, 16,763

Zentner, A. R., Berlind, A. A., Bullock, J. S., Kravtsov, A. V., \& Wechsler, R. H. 2005, ApJ, 624, 505

Zentner, A. R., \& Bullock, J. S. 2003, ApJ, 598, 49

Zheng, Z., Coil, A. L., \& Zehavi, I. 2007, ApJ, 667, 760

Zibetti, S., \& Ferguson, A. M. N. 2004, MNRAS, 352, L6

Zibetti, S., White, S. D. M., Schneider, D. P., \& Brinkmann, J. 2005, MNRAS, 358, 949 